\title{
THEORY OF DIGITAL FILTER BANKS REALIZED VIA MULTIVARIATE EMPIRICAL MODE DECOMPOSITION
}

\author{
MIN-SUNG $\mathrm{KOH}^{*}$ \\ Department of Engineering and Design \\ Eastern Washington University \\ Cheney, WA 99004, USA \\ mkoh@ewu.edu \\ DANILO P. MANDIC ${ }^{\dagger}$ and ANTHONY G. CONSTANTINIDES ${ }^{\ddagger}$ \\ Department of Electrical and Electronic Engineering \\ Imperial College London, London SW7 $2 A Z, U K$ \\ †.mandic@imperial.ac.uk \\ ${ }_{\ddagger}$ a.constantinides@ic.ac.uk
}

Received 19 October 2013

Accepted 12 November 2013

Published 27 January 2014

\begin{abstract}
Undecimated and decimated multivariate empirical mode decomposition filter banks (MEMDFBs) are introduced in order to incorporate MEMD equipped with downsampling into any arbitrary tree structure and provide flexibility in the choice of frequency bands. Undecimated MEMDFBs show the same results as those of original MEMD for an octave tree structure. Since the exact cut-off frequencies of MEMD are not known (i.e. due to data-driven decomposition), employing just simple downsampling in MEMD might cause aliasing. However, decimated MEMDFBs in this paper achieve perfect reconstruction with aliasing cancelled for any arbitrary tree. Applications of decimated/undecimated MEMDFBs for speech/audio and image signals are also included. Since decimated MEMDFBs can be applied into any arbitrary tree structure, this extends into MEMD packets. Arbitrary tree structures in decimated MEMDFBs also lead to more diverse choices in frequency bands for various multivariate applications requiring decimations.
\end{abstract}

Keywords: Multivariate empirical mode decomposition; empirical mode decomposition; multivariate signal analysis; intrinsic mode function; multiscale analysis; filter banks.

\section{Introduction}

Although many real-world signals are neither stationary nor linear, many traditional signal processing techniques are based on linear time invariant (LTI) assumption. Although some signals are close enough to LTI property when considered over

\footnotetext{
${ }^{*}$ Corresponding author.
} 
short-time frames, in many cases, the requirement of LTI is far rigid. To handle real-world signals without stationarity and linearity assumptions, nontraditional techniques are required. A new signal processing technique without stationary and linear assumptions was introduced in Huang et al. [1998] and termed empirical mode decomposition (EMD). The EMD decomposes a signal into intrinsic mode functions (IMFs) and a residual, where IMFs are amplitude modulation (AM)/frequency modulation (FM) like signals and the residual represents the trend within the original signal. The 1st IMF is obtained by subtracting mean envelope from the original signal, where the mean envelope is made by arithmetic average operation of upper envelope and lower envelope. Upper and lower envelopes are respectively calculated by interpolation of detected local maxima and minima points. Residual after taking 1st IMF is obtained by subtraction of the 1st IMF from the original signal, where the residual must be monotonic function or satisfies stop criterion [Huang et al. (1998)]. Otherwise, these steps, called the sifting process must be repeated. To obtain the next IMF, the same processes are applied but the residual is now used as the original signal. EMD is a fully data-driven decomposition, without requiring any traditional filters, it is a very useful signal processing tool for nonstationary and nonlinear signals and has been applied to many different applications such as seismic signal analysis [Zhang et al. (2003)], EEG signal analysis [Park et al. (2011)], signal denoising [Omitaoumu et al. (2011)], speech enhancement [Chatlani and Soraghan (2012)], etc. to name only a few.

Traditional filters with LTI assumption were extended into filter banks, which are composed of low pass filter, band-pass filters, and high pass filter. Filter banks generate subband signals which might have different various signal characteristics, e.g. different speech formants, image edges, etc. A tutorial for traditional filter banks and subband approach can be found in Vaidyanathan [1993] and Vetterli and Kovacevic [1995]. Most successful (and popular) traditional filter banks are filter banks generated by wavelets and short-time Fourier transform (STFT). Those traditional filter banks have been used in many applications such as speech/audio, image, video signal processing [Vaidyanathan (1993); Vetterli and Kovacevic (1995)], and they provide a useful analysis tool to represent a signal in the time-frequency $(\mathrm{T}-\mathrm{F})$ plane. Hence, in order to have a similar $\mathrm{T}-\mathrm{F}$ analysis tool-like traditional filter banks, EMD has also been expected to extend into filter banks. However, the most challenging task to extend EMD into filter banks is incorporating down/up samplings because down/up samplings are fundamental building blocks in filter banks design for multirate processing of speech/audio, image, video signals, etc. Since there are no traditional filters involved in EMD, any simple down/up sampling process ends up with failure of perfect reconstruction. To resolve the problem, downsampling with even and odd indices is introduced for $1 \mathrm{D} / 2 \mathrm{D}$ signals and named as EMD filter banks (EMDFBs) in Koh and Rodriguez-Marek [2013a, 2013b, 2013c]. The EMDFBs provide perfect reconstruction without any traditional filters, while keeping all desirable characteristics of EMD. While traditional filter banks built by wavelets and STFT result in a fixed T-F plane, EMDFBs lead to an adaptive 
data-driven T-F plane without any fixed filters. Compared with traditional filter banks, EMDFBs show several advantages: (i) Nonstationary and nonlinear signals can be handled without approximate assumptions. (ii) Adaptive data-driven subband signals are available, generating an adaptive data-driven $\mathrm{T}-\mathrm{F}$ plane. (iii) There are no filter delays because no filters are used. Hence, the additional step required for the synchronization of each subband signal is not needed.

Although EMD has been applied successfully to various applications [e.g. Zhang et al. (2003); Park et al. (2011); Omitaoumu et al. (2011); Chatlani and Soraghan (2012)] to deal with nonstationary and nonlinear signals, it typically suffers from mode-mixing [ $\mathrm{Wu}$ and Huang (2009)] and aliasing problems. The mode-mixing problem refers to one mode appearing in different IMFs and the aliasing problem refers to problems caused by extrema sampling and interpolations [Mandic et al. (2013); Rehman et al. (2013)]. In addition, the original EMD in Huang et al. [1998] is not applicable to multichannel speech signals because an interpolation to make upper and lower envelops is not clearly defined for multichannel signals. To extend the same idea of original EMD to multivariate (or multichannel) signals, multivariate EMD (MEMD) and noise-aided MEMD (NA-MEMD) were developed in Mandic et al. [2013], Rehman and Mandic [2010], and Rehman et al. [2013], which project data onto hyperspheres to find the multidimensional envelopes. They show good results in signal decompositions and on $\mathrm{T}-\mathrm{F}$ analysis, which enables better interpretation of physical meanings in each decomposed signal. Mode-mixing and aliasing problems are also alleviated through MEMD, as all IMFs are well aligned [Mandic et al. (2013); Rehman and Mandic (2010); Rehman et al. (2013)]. However, the theory of filter banks based on MEMD is still lacking. Hence, similar idea to Koh and Rodriguez-Marek [2013a, 2013b, 2013c] is adopted in this paper to design MEMD filter banks (MEMDFBs), resulting in an adaptive data-derived $\mathrm{T}-\mathrm{F}$ plane. Moreover, since MEMDFBs is applicable into any arbitrary tree structure, it will be extended in this paper into MEMD and/or NA-MEMD packets coined by similar name to wavelet packets. The paper is organized as follows. The MEMD and NA-MEMD introduced in Mandic et al. [2013], Rehman and Mandic [2010], and Rehman et al. [2013] are briefly summarized in Sec. 2. Undecimated and decimated MEMDFBs are introduced respectively in Secs. 3 and 4. Section 5 shows some applications of undecimated/decimated MEMDFBs into multichannel signals, speech, and image signals. Conclusions are given in Sec. 6.

\section{MEMD and NA-MEMD}

Although EMD in Huang et al. [1998] provides a new way to deal with nonstationary and nonlinear signals, the mode-mixing and mode alignment problems need to be resolved when the traditional EMD is considered for multichannel applications. As briefly mentioned, MEMD resolved the concepts of local maxima and minima in a multivariate setting by utilizing a projection concept into $(N-1)$ spheres, where $N$ is the number of variables (or channels) [Mandic et al. (2013); 
Rehman and Mandic (2010)]. To identify local maxima and minima points in multidimensional signals, MEMD projects a signal along multiple direction vectors. Then, it interpolates identified local maxima and minima points in each directional projection to make each directional envelope curves. The mean envelope of a multidimensional signal is obtained by an approximated integral of all interpolated envelope curves. In this process, each directional vector is chosen to be spaced uniformly on the same multidimensional sphere in order to have better accuracy of the approximated integral. Uniformly spaced directional vectors are obtained by a special technique using the Hammersley sequence [Rehman and Mandic (2010)]. The MEMD extracts well common rotational modes buried in a multidimensional signal with good mode alignment property, where rotational modes in MEMD are corresponding to AM/FM oscillating modes in EMD. MEMD, as summarized in Table 1, is shown in Mandic et al. [2013] and Rehman and Mandic [2010].

Since traditional EMD needs a sufficient number of extrema points for a sifting process, a signal without enough extrema (e.g. impulse signal having only one peak) is not decomposed successfully. To resolve the issue, ensemble EMD (EEMD) is developed in Wu and Huang [2009] and the EEMD adds zero-mean white Gaussian noises to a signal to be decomposed at the beginning of EEMD so that it can have enough extrema. Then, each decomposed IMF by EEMD from the noisy original signal is ensemble averaged so that any effects from remaining noise in IMFs due to the included noise can be removed. Since zero-mean white Gaussian noise can be averaged out statistically through the ensemble mean for a large ensemble number, this helps to resolve the problem. However, since EEMD needs a large ensemble number to cancel out the effect of noise, it has a disadvantage in reconstructing original signal from the decomposed IMFs if a sufficient large ensemble mean is not used. Within NA-MEMD [Rehman et al. (2013)], noise is not directly added to original signal but is contained in other channel signals in MEMD. Therefore, NA-MEMD uses zero-mean white Gaussian noises in separate channels (2 or 3 channels) from the original signal and subsequently performs MEMD. In this paper,

Table 1. Algorithm for MEMD [Mandic et al. (2013); Rehman and Mandic (2010)].

1. Generate a $V$-point Hammersley sequence used for uniformly sampling a $N$-dimensional sphere.

2. Calculate the projections $q_{\theta_{v}}(t)$ of the original signal $s(t)$ along the direction vector $x_{\theta_{v}}$, for $v=1,2, \ldots, V$ to give the set of projections $\left\{q_{\theta_{v}}(t)\right\}_{v=1}^{V}$.

3. Find the time instants $\left\{t_{\theta_{v}}^{i}\right\}_{v=1}^{V}$ corresponding to the maxima of the set of projected signals $\left\{q_{\theta_{v}}(t)\right\}_{v=1}^{V}$.

4. Interpolate $\left[t_{\theta_{v}}^{i}, s\left(t_{\theta_{v}}^{i}\right)\right]$ to obtain the multivariate envelope curves $\left\{e_{\theta_{v}}(t)\right\}_{v=1}^{V}$.

5. Calculate the mean of the $V$ mulitidimensional envelopes:

$$
m(t)=\frac{1}{V} \sum_{v=1}^{V} e_{\theta_{v}}(t)
$$

6. Extract the "detail" $d(t)=s(t)-m(t)$. If $d(t)$ fulfills some stoppage criterion for a multivariate IMF, apply the above procedure to $s(t)-d(t)$, else, repeat for $d(t)$. 
NA-MEMD is used without ensemble mean because noise is not directly added to a signal. Hence, the NA-MEMD algorithm is the same as the MEMD but additional 2 or 3 channels are included at the beginning of MEMD as shown in Mandic et al. [2013] and Rehman et al. [2013].

\section{Undecimated MEMDFBs}

In this paper, undecimated MEMDFBs are designed by extending the concept of undecimated EMDFBs in Koh and Rodriguez-Marek [2013b]. To make a filter bank structure with MEMD, just one IMF and one residual are considered at each decomposition step, like EMDFBs in Koh and Rodriguez-Marek [2013a, 2013b]. Since one IMF (usually the first IMF) and the residual are corresponding to a high frequency and a low frequency respectively, those two signals are used for a filter bank structure in this paper. Hence, the concept of Koh and Rodriguez-Marek [2013a, 2013b] is easily extended into MEMD by applying it to each variable (or channel) in MEMD. Each MEMD in MEMDFBs produces multiple first-IMFs and multiple residuals for all $N$ variables. Then, as an example, an undecimated MEMDFB can be obtained as Fig. 1 for decomposition level of 2, where $X_{00} \in R^{L \times N}$ is the original multivariate signal for $N$ variables with $L$ data points each. In Fig. 1, the node signals, $X_{10}$ and $X_{11}$, are corresponding respectively to one residual and one IMF of the mother node signal, $X_{00}$. Figure 1 is similar to those in Koh and Rodriguez-Marek [2013b] except for the use of MEMD in order to accommodate multivariate signals.

Since MEMD in Mandic et al. [2013] and Rehman and Mandic [2010] shows a good mode-splitting property at each IMF, an octave tree structure in undecimated MEMDFBs shows the same results as those of MEMD. For instance, consider an octave tree structure at the decomposition level of 2 (i.e. $(2,0),(2,1)$, and $(1,1)$

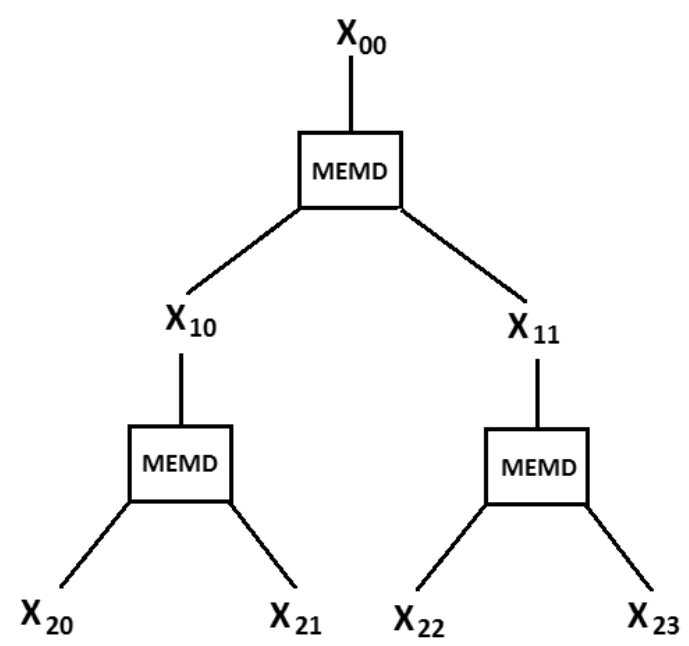

Fig. 1. Undecimated MEMD analysis filter banks of full binary tree at decomposition level of 2 . 
nodes only to be an octave tree in Fig. 1), then the 1st, 2nd IMFs, and residual are respectively corresponding to $(1,1),(2,1)$, and $(2,0)$ node signals. An example of an octave tree is shown in Fig. 2(b), which is the results of undecimated EMDFB with an octave tree having $(8,0),(8,1),(7,1),(6,1),(5,1),(4,1),(3,1),(2,1)$, and
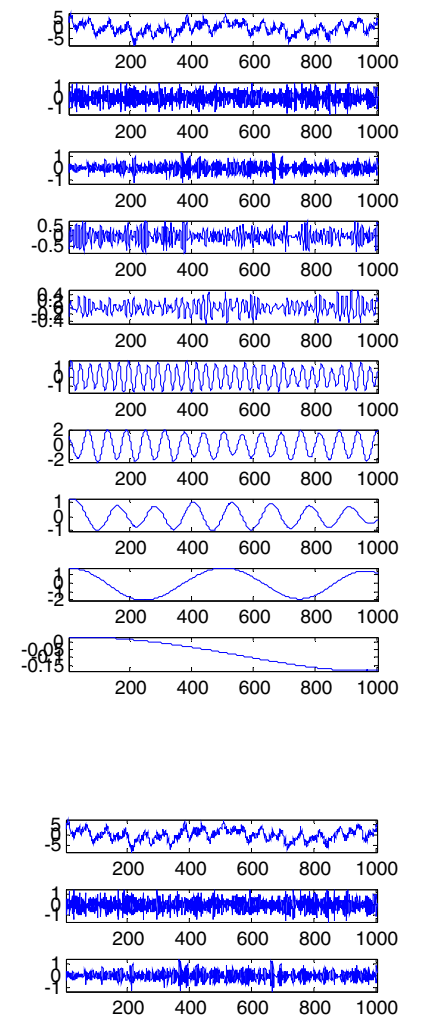

0.5
-0.5 $\begin{array}{lllll}200 & 400 & 600 & 800 & 1000\end{array}$

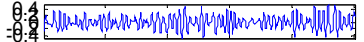
$\begin{array}{lllll}200 & 400 & 600 & 800 & 1000\end{array}$ -

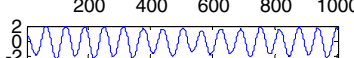

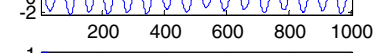
$0 \frac{1}{200 \quad 400600 \quad 800 \quad 1000}$

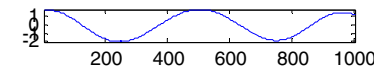

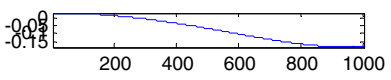

$=8$. $200 \quad 400 \quad 600 \quad 800 \quad 1000$

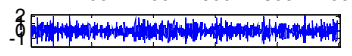

$\begin{array}{lllll}200 & 400 & 600 & 800 & 1000\end{array}$

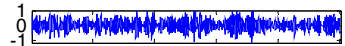
$\begin{array}{lllll}200 & 400 & 600 & 800 & 1000\end{array}$

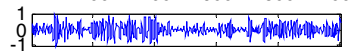
$200 \quad 400 \quad 600 \quad 800 \quad 1000$

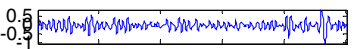
$200 \quad 400 \quad 600 \quad 800 \quad 1000$

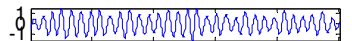
$\begin{array}{lllll}200 & 400 & 600 & 800 & 1000\end{array}$

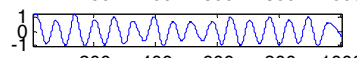
$200 \quad 400 \quad 600 \quad 800 \quad 1000$
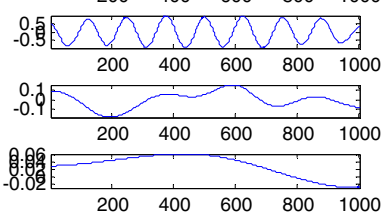

(a)

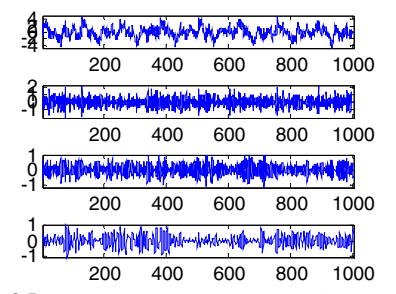

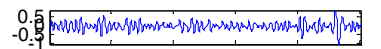

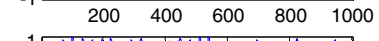

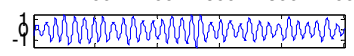
$\begin{array}{lllll}200 & 400 & 600 & 800 & 1000\end{array}$ -1 uninuminuna

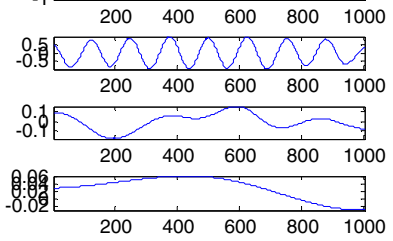

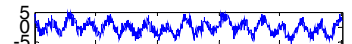

$\begin{array}{llllll}-5 & 200 & 400 & 600 & 800 & 1000\end{array}$ $\int_{-2}^{2}$ (s) $\begin{array}{lllll}200 & 400 & 600 & 800 & 1000\end{array}$

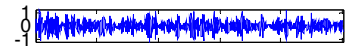
$\begin{array}{lllll}200 & 400 & 600 & 800 & 1000\end{array}$ 0.5
-0.9 $\begin{array}{lllll}200 & 400 & 600 & 800 & 1000\end{array}$

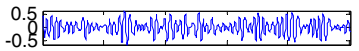
$\begin{array}{rrrrr}200 & 400 & 600 & 800 & 1000\end{array}$

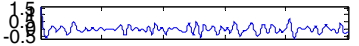

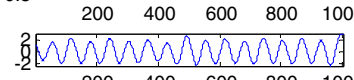
$\begin{array}{lllll}200 \quad 400 \quad 600 & 800 \quad 1000\end{array}$ 0.0 \begin{tabular}{llllll}
200 & 400 & 600 & 800 & 1000 \\
\hline
\end{tabular} \begin{tabular}{lllllll}
0.3 & & & & & & \\
\hline
\end{tabular}

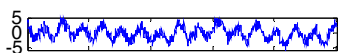
$\begin{array}{lllll}200 & 400 & 600 & 800 & 1000\end{array}$

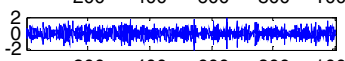
$\begin{array}{lllll}200 & 400 & 600 & 800 & 1000\end{array}$

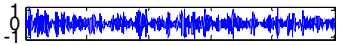
$\begin{array}{lllll}200 & 400 & 600 & 800 & 1000\end{array}$ 0.5 . $\begin{array}{lllll}200 & 400 & 600 & 800 & 1000\end{array}$

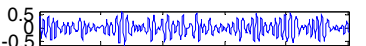
$\begin{array}{lllll}200 & 400 & 600 & 800 \quad 1000\end{array}$

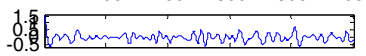
$\begin{array}{lllll}200 & 400 & 600 \quad 800 \quad 1000\end{array}$

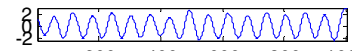
$\frac{100 \quad 400 \quad 600 \quad 800}{1000}$

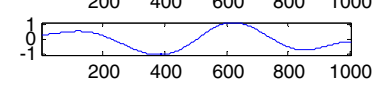

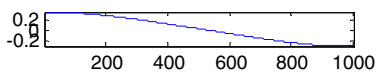

(b)

Fig. 2. Comparison of undecimated MEMDFB with MEMD in Mandic et al. (2013) and Rehman and Mandic (2010). (a) Results of MEMD in Mandic et al. [2013] and Rehman and Mandic [2010] and (b) undecimated MEMDFB results for an octave tree. 
$(1,1)$ end-nodes. Figure 2(a) is the results of MEMD developed in Mandic et al. [2013] and Rehman and Mandic [2010] for a comparison. Notice that the first row of Figs. 2(a) and 2(b) contains the given signals for three channels. In Fig. 2(a), the 2nd and the last rows are for the 1st IMF and residual, respectively. In Fig. 2(b), 2nd, $3 \mathrm{rd}, 4$ th and the last rows are respectively for $(1,1),(2,1),(3,1)$, and $(8,0)$ node signals. All other IMFs and node signals are listed in the same manner in Fig. 2.

For undecimated MEMFBs, other further decompositions are effectively the same, with an octave tree structure, because of the good mode-splitting property of MEMD. In other words, even with a further decomposition of $(1,1)$ node signal into $(2,2)$ and $(2,3)$ nodes, the undecimated MEMDFB shows no residual at the $(2,2)$ node, and the same for the $(1,1)$ node signal at $(2,3)$ node. However, this is not true for decimated EMDFBs, which will be explained in next section, this is because downsamplings make it different from the results of original MEMD.

\section{Decimated MEMDFBs}

Although the original EMD for a single variable in Huang et al. [1998] has many advantages in dealing with real-world signals, it decomposes a signal only by a fixed tree structure, which is an octave tree structure. This is because only the residual (corresponding to a low pass signal) is further decomposed. Hence, the so-obtained IMFs are not further decomposed by the original EMD although there might be a signal to be further decomposed in an IMF [i.e. mode-mixing problem addressed in Mandic et al. (2013)]. Moreover, all decomposed signals have the same length of data because there is no downsampling, which is used in traditional filter banks. Downsampling is a key element in modern filter banks to reduce data obtained by various decompositions. However, it is not straightforward to incorporate downsamplings into (M)EMD because traditional filters satisfying perfect reconstruction property (e.g. quadrature mirror filters, QMFs) are not available in (M)EMD. To overcome those drawbacks of original EMD, EMDFBs are introduced in Koh and Rodriguez-Marek [2013a, 2013b, 2013c] as an extension of Huang et al. [1998] into filter bank theory. EMDFBs introduced in Koh and Rodriguez-Marek [2013a, 2013b, 2013c] incorporate downsamplings to reduce decomposed data and it is applicable into any arbitrary tree structure so that chosen frequency bands are flexible to create any nonuniform filter banks. Figure 3 shows one stage of analysis and synthesis filter banks in decimated EMDFBs. Figure $3\left(\right.$ a) decomposes a signal, $X_{i j}$, into one IMF denoted by $X_{i+1,2 j+1}$ and one residual denoted by $X_{i+1,2 j}$, where $X_{i+1,2 j}$ and $X_{i+1,2 j+1}$ are down sampled by 2 . The signal denoted by $\Delta_{i+1, j}$ is an error signal between the estimated even-indexed signal and true even-indexed signal, where the estimated even-indexed signal is obtained by downsampling of the interpolated signal of the odd-indexed residual denoted by $R_{o}$. Figure $1(\mathrm{~b})$ is a counterpart of (a) to recover the original signal $X_{i j}$, where the interpolation must be the same interpolation used in the analysis stage of Fig. 1(a). The delay operators, denoted by $Z^{-1}$ in Fig. 1(a), take even indexed samples and the operator denoted by $Z$ in 


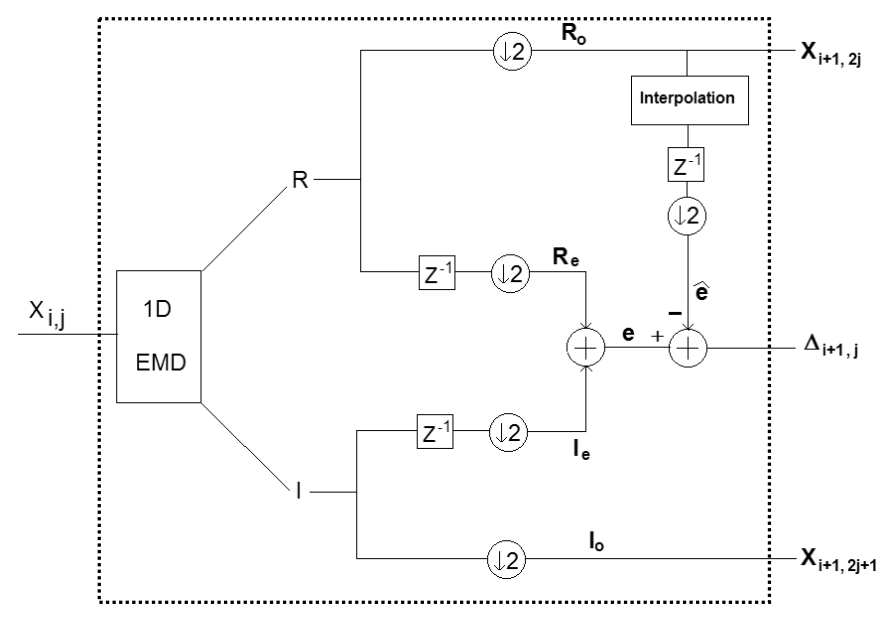

(a)

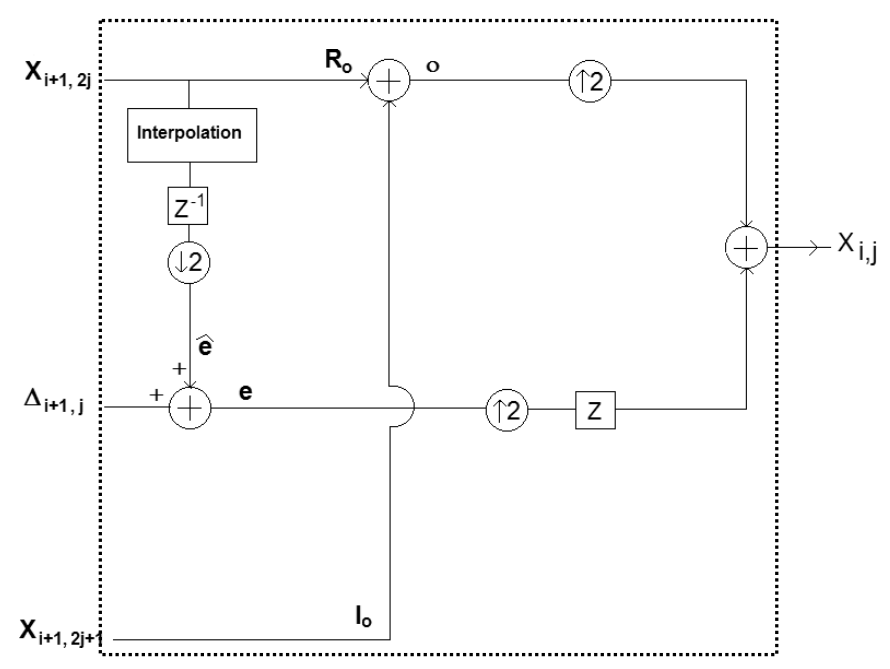

(b)

Fig. 3. Single stage of the decimated EMD analysis and synthesis filter banks [Koh and Rodriguez-Marek (2013a, 2013b)]. (a) One analysis stage and (b) one synthesis stage

Fig. 1(b) moves back the even indexed samples into the original positions [Koh and Rodriguez-Marek (2013a, 2013b)].

Figure 3 guarantees a perfect reconstruction for any arbitrary EMDFBs, as proved in Koh and Rodriguez-Marek [2013a]. Since Fig. 3 is extended into decimated MEMDFBs for multichannel data in this paper, we next state the theorem of perfect reconstruction for EMDFBs, where a detailed proof is given in Koh and Rodriguez-Marek [2013a]. 
Theorem 1. (Perfect reconstruction of EMDFBs) [Koh and Rodriguez-Marek (2013a)]: The decimated EMD filter banks in Figs. 3(a) and 3(b) have perfect reconstruction and cancel aliasing, provided the same interpolation technique is used for analysis and synthesis.

Since MEMD has a property of filter banks as shown in Rehman and Mandic [2011], MEMD opens some possibilities to be used as a new filter bank with downsamplings for nonlinear and nonstationary signals. Decimated MEMDFBs introduced in this section apply to Fig. 3 in multichannel settings and incorporate downsamplings. Hence, a similar idea to Koh and Rodriguez-Marek [2013a, 2013b] is used as shown in Fig. 4, where Fig. 4 is an extended version of Koh and Rodriguez-Marek [2013a, 2013b] for $N$ variables (or channels). In Fig. 4, suppose a $X_{i, j} \in R^{L \times N}$, then $X_{i+1,2 j}, \Delta_{i+1, j}$, and $X_{i+1,2 j+1} \in R^{\frac{L}{2} \times N}$ are respectively corresponding to down sampled residuals, errors, and IMFs of all $N$ channels for the mother node signal, $X_{i, j}$.

As shown in Fig. 4, each stage produces three outputs, which are one IMF, residual, and error signals. One stage in Fig. 4 is connected into lower nodes for a given arbitrary tree, as shown in Fig. 5 with a decomposition level of 2. Figure 5 is the same as for a single variable case shown in Koh and Rodriguez-Marek [2013b] but note that the data sizes are different for multivariable cases. For instance, all node signals at the decomposition level 2 have the dimension of $\frac{L}{4} \times N$ for a given $X_{00} \in R^{L \times N}$ (i.e. $X_{20}, X_{21}, X_{22}, X_{23}, \Delta_{20}$, and $\Delta_{21} \in R^{\frac{L}{4} \times N}$ ) because two downsampling steps are applied into each node path at the first and the second decomposition levels.

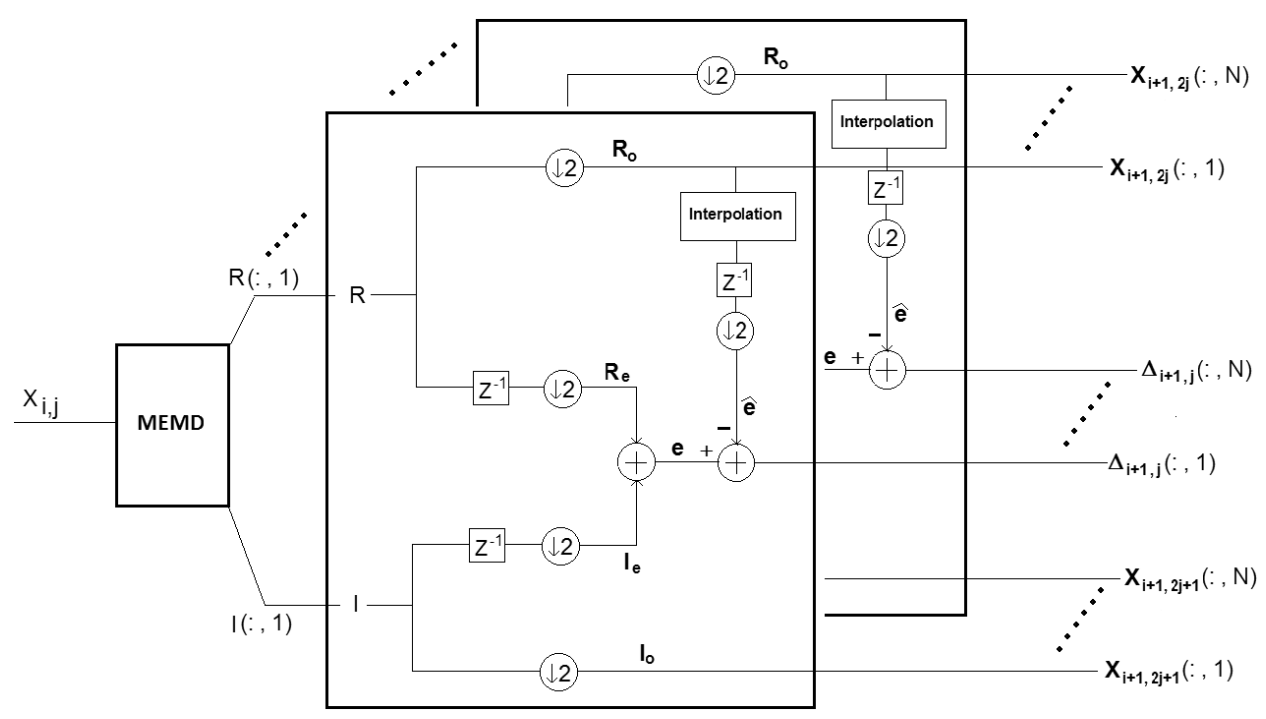

Fig. 4. One stage of analysis filter banks for decimated MEMDFBs (at the $i$ th decomposition level). 


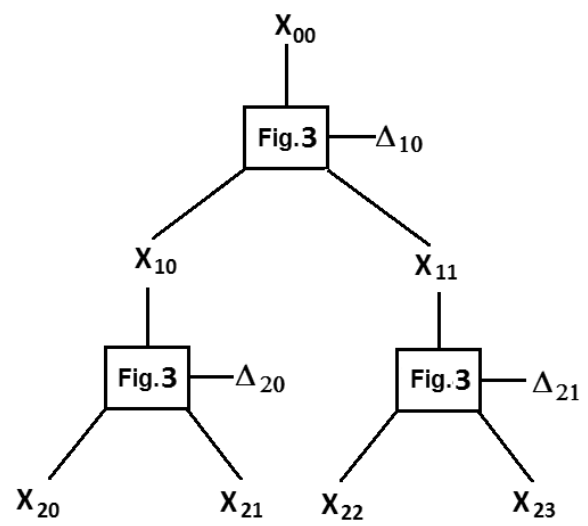

Fig. 5. Decimated MEMD analysis filter banks of a full binary tree at the decomposition level of 2 .

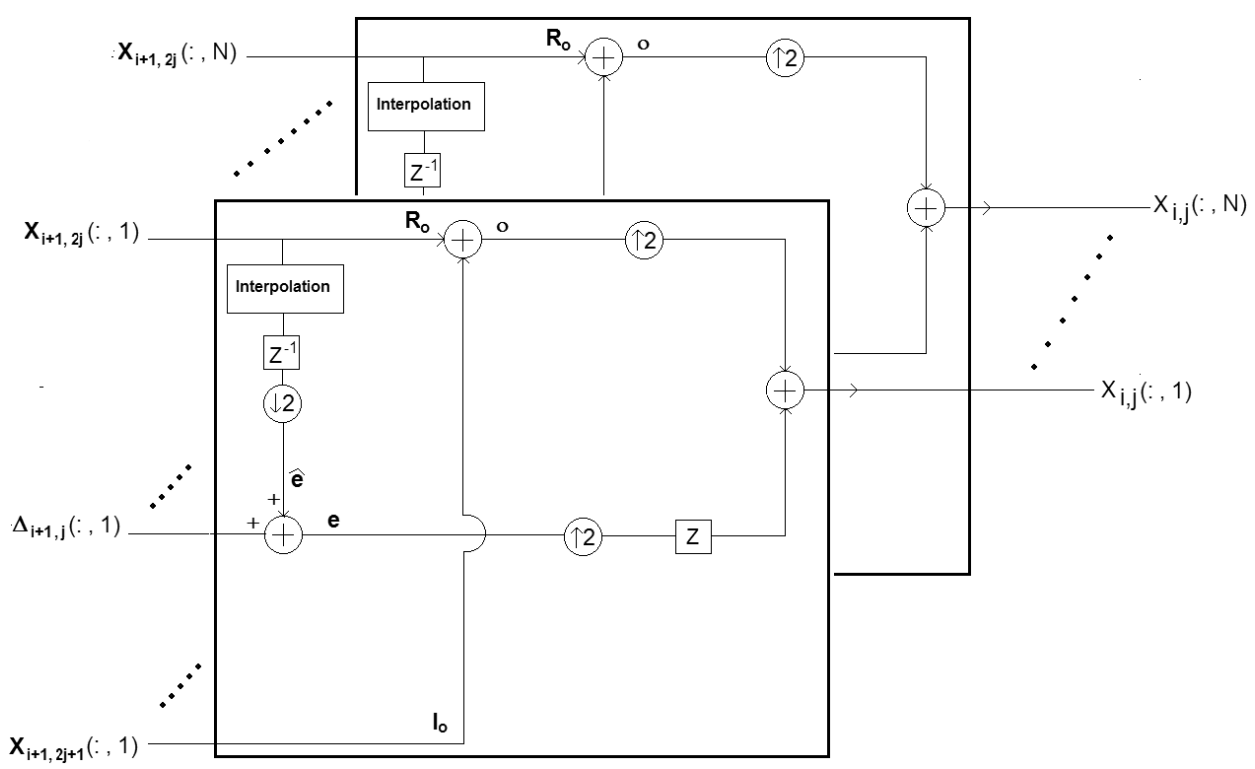

Fig. 6. One stage of synthesis filter banks for decimated MEMDFBs (at the $i$ th decomposition level).

To achieve perfect reconstruction, synthesis filter banks are also required for each node signal. One stage of the synthesis filter bank is shown in Fig. 6, which is an extension of Fig. 3 to the multivariate case. As mentioned, a single variable version of Figs. 4 and 6 guarantees perfect reconstruction with aliasing cancelled through Theorem 1. Since decimated MEMDFBs are an extension of the single variable case, Figs. 4 and 6 also guarantee a perfect reconstruction, with aliasing cancelled. To recover the given original signal, Fig. 6 is applied with a given tree structure starting from the end-nodes up to $(0,0)$ node. The perfect reconstruction 
ability of MEMDFBs is summarized in the following corollary. Since the Corollary is an extension of Theorem 1 into multichannel cases, the proof of Corollary is a simple extension of the proof in Koh and Rodriguez-Marek [2013a] applied to each channel.

Corollary 1. (Perfect reconstruction of MEMDFBs): Decimated analysis and synthesis MEMDFBs of Figs. 4 and 6 respectively form a perfect reconstruction with aliasing cancelled.

The data reduction ratio (DRR) of decimated MEMDFBs compared with traditional MEMD [Mandic et al. (2013); Rehman and Mandic (2010)] for one channel is the same, with DRR $=\frac{(P+1) L}{L\left(2-\frac{1}{2^{P}}\right)}=\frac{P+1}{\left(2-\frac{1}{2^{P}}\right)}$ as shown in Koh and Rodriguez-Marek [2013a], where $P$ is a given decomposition level and $L$ is data length of each channel. The numerator, $(P+1) L$, reflects the intrinsic octave tree structure of MEMD in one channel. In other words, if $P$ of IMFs are obtained by MEMD, with one residual for data length of $L$, then traditional MEMD has $(P+1) L$ decomposed data for a single channel. For the same octave tree, decimated MEMDFBs have the denominator of $L\left(2-\frac{1}{2^{P}}\right)$, which is obtained by adding all lengths of node signals and error node signals for the same $P$ decomposition level with one channel case [Koh and Rodriguez-Marek (2013a)]. Hence, DRR for all MEMD channels is obtained by $\mathrm{DRR}=\frac{(P+1) N}{\left(2-\frac{1}{2^{P}}\right)}$ for an $N$-channel case. For instance, assume that traditional MEMDs have seven IMFs and one residual (i.e. $P=7$ ) with eight-channels (i.e. $N=8$ ), then $\mathrm{DRR} \approx 32$. In other words, decimated MEMDFBs have 32 times less decomposed data compared with traditional MEMD.

\section{Applications of MEMDFBs to Multivariate, Image, and Speech Signals}

The MEMDFBs, explained in previous sections, are now applied to multivariate signals, one-dimensional signals (e.g. speech and audio signals), and two-dimensional signals (e.g. images). Since speech and images are usually one-channel applications, revised MEMDFBs are required for those one channel signals, and the revisions are explained below.

\subsection{Multivariate signals}

Undecimated and decimated MEMDFBs are first applied to real-world signals. Figure 7(a) shows all decomposed signals by a decimated MEMDFB for a hexavariate real world taichi dataset with a tree having end-nodes of $(6,0),(6,1),(5,1)$, $(4,1),(3,1),(2,1)$, and $(1,1)$. The first row of Fig. $7(\mathrm{a})$ is corresponding to the $(1,1)$ node signal and the last row of Fig. 7 (a) is corresponding to the $(6,0)$ node signal. In Fig. 7(a), note that the decomposed signal lengths are getting reduced by a factor of 2, as the decomposition level increases. Also, note that the delta signals in Fig. 4, which are required to achieve perfect reconstruction for decimated MEMDFBs, are 


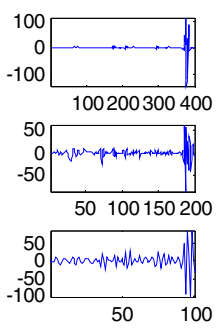

romm
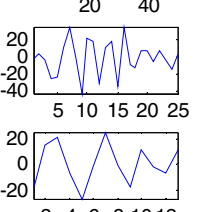

$246 \quad 8 \quad 1012$

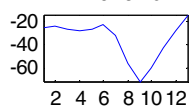

24681012
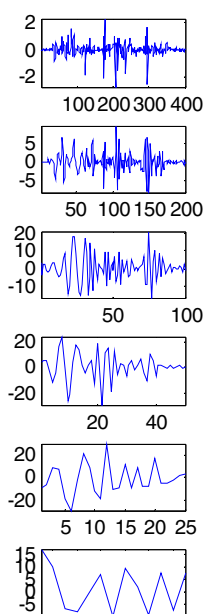

24681012

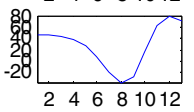

$2 \begin{array}{lllll}4 & 6 & 8 & 1012\end{array}$
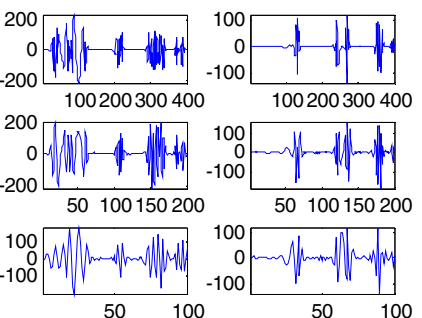

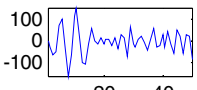
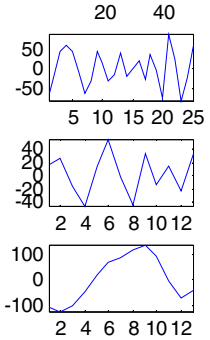

(a)

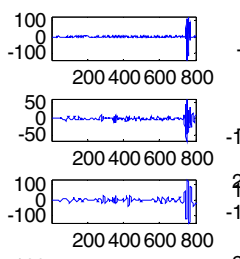

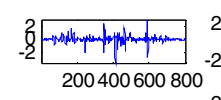

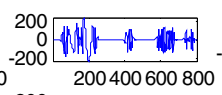

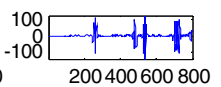

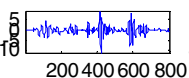

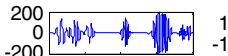

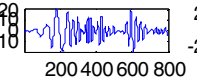

200400600800

-50 ringurin

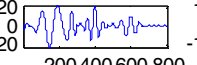

200400600800

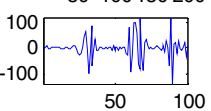

$\frac{50}{20}$
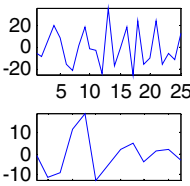

$\begin{array}{llllll}2 & 4 & 6 & 8 & 1012\end{array}$
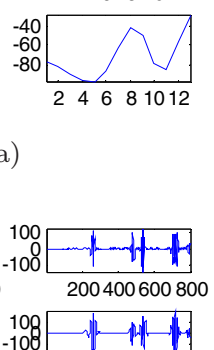

200 a)

200400600800

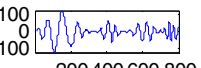

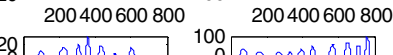

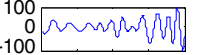

200400600800

$\frac{200400600800}{200}$

200400600800

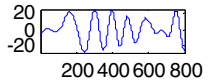

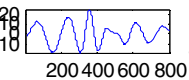

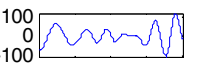

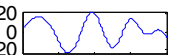

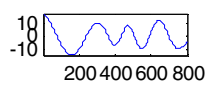

200400600800

200400600800

200400600800
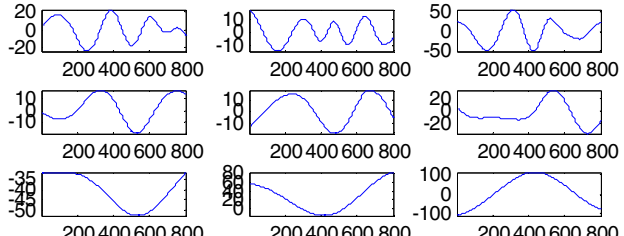

200400600800

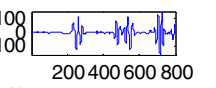

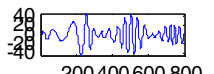

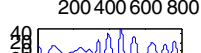

:

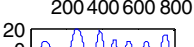

02 /Mn

200400600800
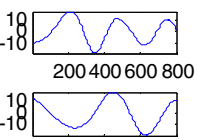

200400600800
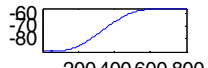
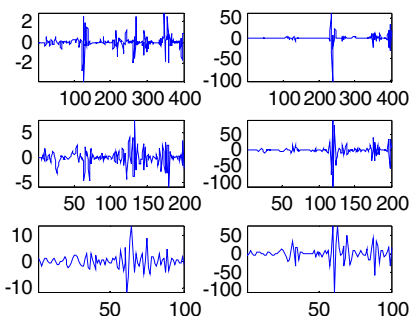

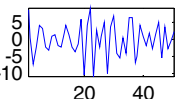

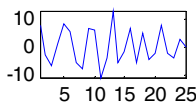

$\frac{50}{0.50 W}$
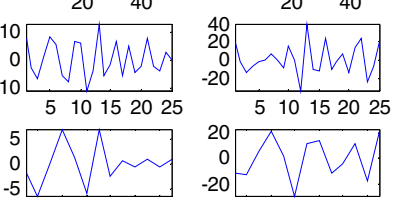

246881012
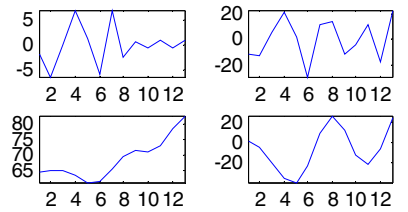

24681012

(b)

Fig. 7. Results of decimated MEMDFBs and original MEMD. (a) Decomposed signals by a decimated MEMDFB and (b) IMFs and residual decomposed by MEMD in Mandic et al. [2013] and Rehman and Mandic [2010]. 
not included in Fig. 7(a). For comparison with MEMD, Fig. 7(b) shows the IMFs decomposed by MEMD in Mandic et al. [2013] and Rehman and Mandic [2010]. The first row of Fig. 7(b) is corresponding to the first IMF decomposed by MEMD.

\subsection{Two-dimensional signals}

The undecimated and decimated MEMDFBs are next applied to an image, where three channels are used. The first, second, and third channels are obtained by scanning an image in column, row, and diagonal directions. To avoid abrupt changes at the end of each column, row, and diagonal, every other column, row, diagonal vectors are flipped. The results of undecimated MEMDFBs is shown in Fig. 8, where an octave tree of $(3,0),(3,1),(2,1)$, and $(1,1)$ end-nodes is used. Since each end-node of Fig. 1, which is for undecimated MEMDFBs, has redundant subimages because of three channels for column, row, and diagonal scanning of an image, the end-node images are arithmetically averaged. For instance, each channel column vector in $X_{11}$ node signal is changed into three channel images of $X_{11, c}^{\mathrm{img}}$, $X_{11, r}^{\mathrm{img}}$, and $X_{11, d}^{\mathrm{img}}$ and those three channel images are arithmetically averaged as $X_{11}^{\mathrm{img}}=\left(X_{11, c}^{\mathrm{img}}+X_{11, r}^{\mathrm{img}}+X_{11, d}^{\mathrm{img}}\right) / 3$. Note that $X_{11}$ is a matrix node signal having three column vectors and $X_{11}^{\mathrm{img}}$ is an image node signal. Hence, any matrix node

$(3,0)$ node image

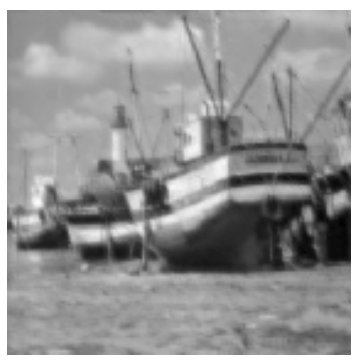

$(2,1)$ node image

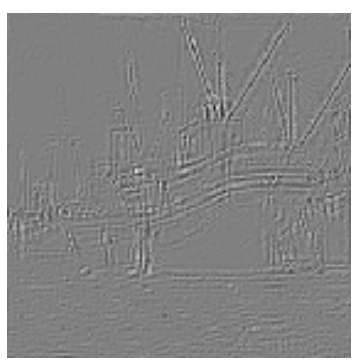

$(3,1)$ node image

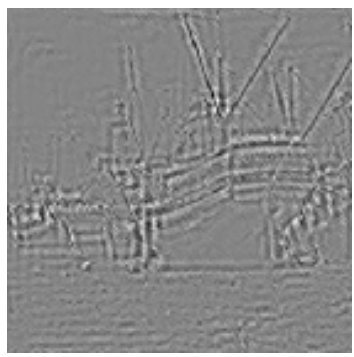

$(1,1)$ node image

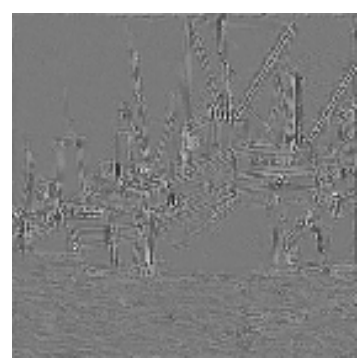

Fig. 8. Decomposed signals by an undecimated MEMDFB. 
signal, $X_{i j}$, is changed into an image node signal, $X_{i j}^{\mathrm{img}}$, by

$$
X_{i j}^{\mathrm{img}}=\left(X_{i j, c}^{\mathrm{img}}+X_{i j, r}^{\mathrm{img}}+X_{i j, d}^{\mathrm{img}}\right) / 3,
$$

All image node signals for the given octave tree of $(3,0),(3,1),(2,1)$, and $(1,1)$ end-nodes are shown in Fig. 8. Since Fig. 8 is the result of undecimated EMDFBs, all node images have the same size.

For decimated MEMDFBs, a similar idea to Koh and Rodriguez-Marek [2013c] is used within MEMD in this paper. Like Koh and Rodriguez-Marek [2013c], downsamplings are applied into row and column directions after changing each channel data into an image at each decomposition level. After downsamplings in the row and column directions, images are put back into column vectors for the next level decompositions. Those ideas are shown in Fig. 9 as block diagrams, where Figs. 9(b) and 9(d) are the same analysis and synthesis filter banks used for 2DEMD in Koh and Rodriguez-Marek [2013c]. In Fig. 9(a), the $V / I$ block implies

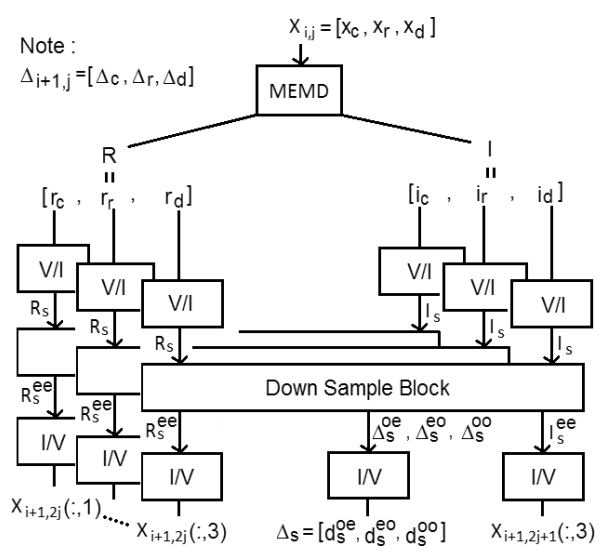

(a)

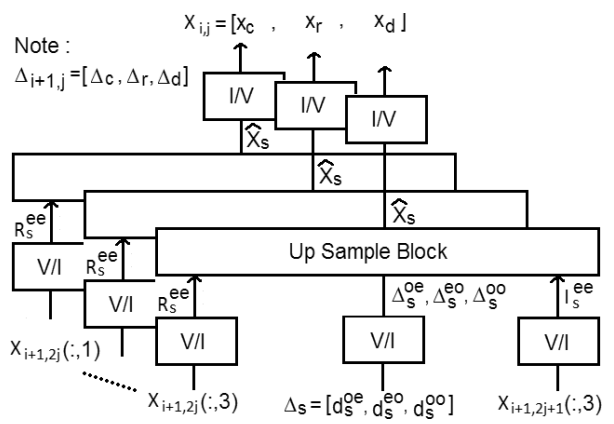

(c)

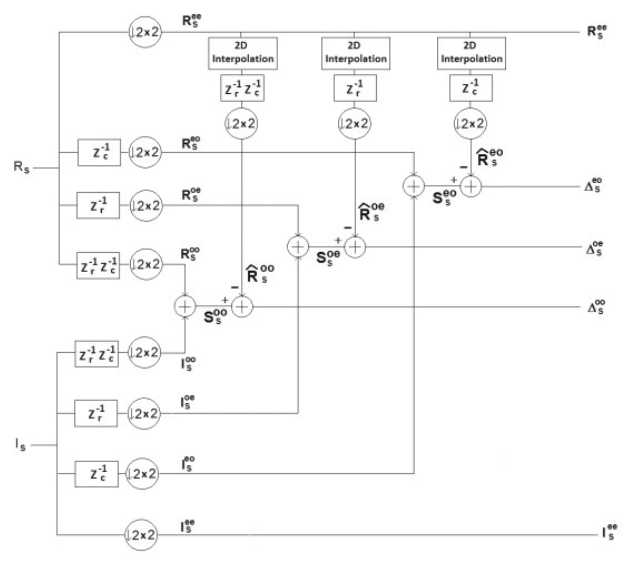

(b)

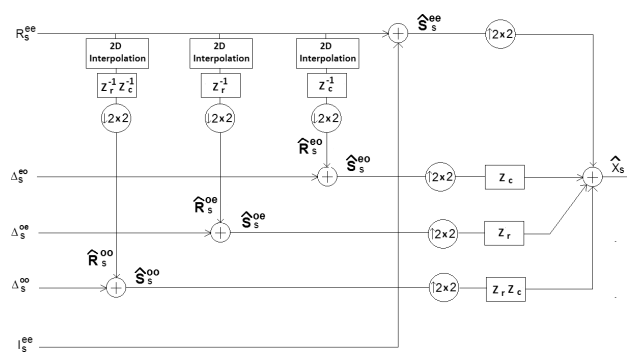

(d)

Fig. 9. Analysis and synthesis MEMFBs for image application only. (a) One stage of analysis MEMDFBs, (b) Downsampling block in (a), (c) One stage of synthesis MEMDFBs and (d) Upsampling block in (c). 
"(column) vector to image", which changes the given column vector into an image. For instance, $X_{i, j}=\left[x_{c}, x_{r}, x_{d}\right] \in R^{L \times 3}$, in Fig. 9(a) is a matrix input, which is scanned from an image in column, row, and diagonal directions, as mentioned. The given matrix input, $X_{i, j}$, is decomposed into two matrices, $R$ and $I$ by MEMD, where $R=\left[r_{c}, r_{r}, r_{d}\right]$ and $I=\left[i_{c}, i_{r}, i_{d}\right]$ having three column vectors each. For instance, $i_{r}$ and $r_{r}$ means respectively the first IMF and residual decomposed by MEMD from the 2nd column vector of $x_{r}$, which is composed by pixels in row direction, of an image. Other column vectors of $r_{c} / i_{c}$ and $r_{d} / i_{d}$ are obtained by the same manner for the image pixels in column and diagonal direction, respectively. Hence, it is obvious that $x_{s}=i_{s}+r_{s}$ for any subscript $s \in\{c, r, d\}$ because of the MEMD nature. The $R_{s}$ in Fig. 9(a) implies a subimage changed respectively from a column vector of $r_{c}, r_{r}$, or $r_{d}$. The block denoted by " $I / V$ " implies "image to (column) vector" by scanning an image in column, row, or diagonal direction. For instance, $X_{i+1,2 j}$ in Fig. 9(a) has three column vectors, which are respectively changed from $R_{c}^{e e}, R_{r}^{e e}$, and $R_{d}^{e e}$ subimages because $R_{s}^{e e}$ implies $R_{c}^{e e}, R_{d}^{e e}$, or $R_{d}^{e e}$ subimage. Since each column of $X_{i+1,2 j}$ is changed from down sampled image in row and column directions as Fig. 9 (b), $X_{i+1,2 j}$ is a $\frac{L}{4} \times 3$ matrix for $X_{i, j} \in R^{L \times 3}$, where $L=M N$ for a given $M \times N$ image. In Figs. 9(a) and 9(b), $\Delta_{s}^{o e}$ implies $\Delta_{c}^{o e}, \Delta_{r}^{o e}$, or $\Delta_{d}^{o e}$, depending on a channel and those are error subimages in (odd, even) indices made by "down sample block (DSB)". The DSB is shown in Fig. 9(b), where the input subimage, $R_{s}$, is a subimage made from $r_{c}, r_{r}$, or $r_{d}$ column vectors in the $R$ matrix. For instance, if two subimages of $R_{d}$ and $I_{d}$ obtained by the $r_{d}$ column vector are applied to Fig. $9(\mathrm{~b})$ for $R_{s}$ and $I_{s}$, then five output images in Fig. 9(b) imply $R_{d}^{e e}, I_{d}^{e e} \Delta_{d}^{o e}, \Delta_{d}^{e o}$, and $\Delta_{d}^{o o}$ subimages. Note that each $\Delta_{s}^{X X}$ subimage in Fig. 9(a) is changed into three column vectors of $\left[d_{s}^{o e}, d_{s}^{e o}, d_{s}^{o o}\right]$ defined by a matrix, $\Delta_{s}$. For instance, $R_{d}$ and $I_{d}$ are applied into DSB inputs, then $\Delta_{s}$ in Fig. 9(a) implies $\Delta_{d}=\left[d_{d}^{o e}, d_{d}^{e o}, d_{d}^{o o}\right] \in R^{\frac{L}{4} \times 3}$ for given $R_{d}, I_{d} \in R^{M \times N}$, where $L=M N$. Hence, the total error node matrix denoted by $\Delta_{i+1, j}=\left[\Delta_{c}, \Delta_{r}, \Delta_{d}\right]$ has a size of $\frac{L}{4} \times 9$. Since Fig. $9(\mathrm{~b})$ is similar in structure to the analysis part in Koh and Rodriguez-Marek [2013c], a detailed explanation is given in Koh and Rodriguez-Marek [2013c] for a single channel.

Figure 9(c) shows one stage of synthesis MEMDFBs, which is the counterpart of Fig. 9(a). Note that in Fig. 9(c) each column vectors in $X_{i+1,2 j}$ is changed respectively into $R_{c}^{e e}, R_{r}^{e e}$, and $R_{d}^{e e}$ subimages but those subimages are denoted by $R_{s}^{e e}$ for a general input belong to subscripts $s \in\{c, r, d\}$, whose notation is also used for "up sample block (USB)" in Fig. 9(d). USB in Fig. 9(c) recovers an image and the recovered image (denoted by $\hat{X}_{s}$ ) is changed into a column vector by " $I / V$ " implying a conversion from an image to a (column) vector. Detailed parts of USB are given in Fig. 9(d), where if $R_{r}^{e e}, I_{r}^{e o}, \Delta_{r}^{o e}, \Delta_{r}^{e o}$, and $\Delta_{r}^{o o}$ subimages are applied into the USB, then the recovered subimage $\hat{X}_{s}$ is changed into a column vector $x_{r}$ in $X_{i, j}$ by $I / V$ block. Figures $9(\mathrm{a})$ and $9(\mathrm{c})$ form perfect reconstruction filter banks, and this is guaranteed by the following Theorem. A detailed proof of the Theorem 2 is given in Appendix A. 
Theorem 2. Decimated MEMDFBs shown in Figs. 9(a) and 9(c) form perfectly reconstructable filter banks, with aliasing cancelling as long as identical $2 D$ interpolation techniques are applied in DSB and USB of the synthesis and analysis respectively.

The results of decimated MEMDFBs using the structure of Fig. 9 are shown in Fig. 10, where (a) shows perfect reconstruction of a decimated MEMDFB. Since

Original image

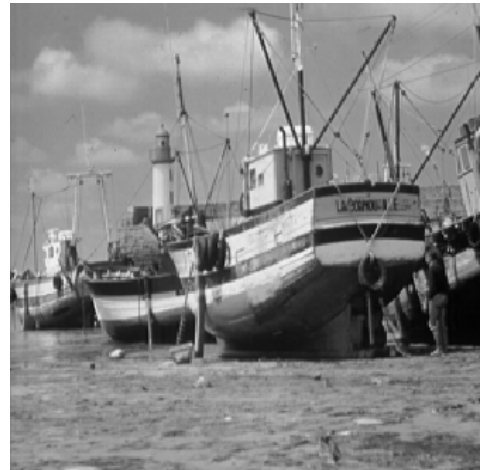

Recovered image by MEMDFBs, SNR=325 [dB]

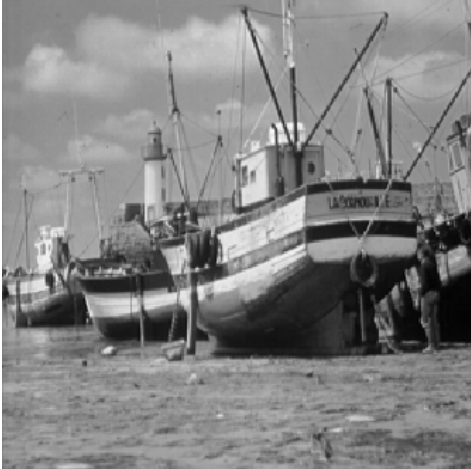

(a)

\begin{tabular}{|c|c|c|c|}
\hline$x_{30}$ & $D_{30}^{\circ e}$ & \multirow{2}{*}{$D_{20}^{0 e}$} & \multirow{3}{*}{$D_{10}^{o e}$} \\
\hline$D_{30}^{e 0}$ & $x_{31}$ & & \\
\hline \multicolumn{2}{|c|}{$\mathrm{D}_{20}^{\mathrm{eo}}$} & $x_{21}$ & \\
\hline \multicolumn{3}{|c|}{$D_{10}^{\mathrm{eo}}$} & $X_{11}$ \\
\hline
\end{tabular}

(b)

Fig. 10. Results applied into an image for a decimated MEMDFB and a comparison with wavelet filter banks. (a) Original image $(256 \times 256)$ and recovered image by a decimated MEMDFB for a tree having $(3,0),(3,1),(2,1)$, and $(1,1)$ end-nodes, (b) Left: Octave tree used for decimated MEMDFBs. Right: Corresponding subimages, (c) Left: Decomposed signals by a decimated MEMDFB for an octave tree of (b), where the corresponding subimages are indexed by right side of (b). Right: Decomposed signal by wavelet filter banks with the same octave tree of (b) and (d) $D_{i j}^{o o}$ images required for decimated MEMDFBs to achieve perfect reconstruction, each one from left is respectively corresponding to $D_{10}^{o o} \in R^{128 \times 128}, D_{20}^{o o} \in R^{64 \times 64}$, and $D_{30}^{o o} \in R^{32 \times 32}$. 

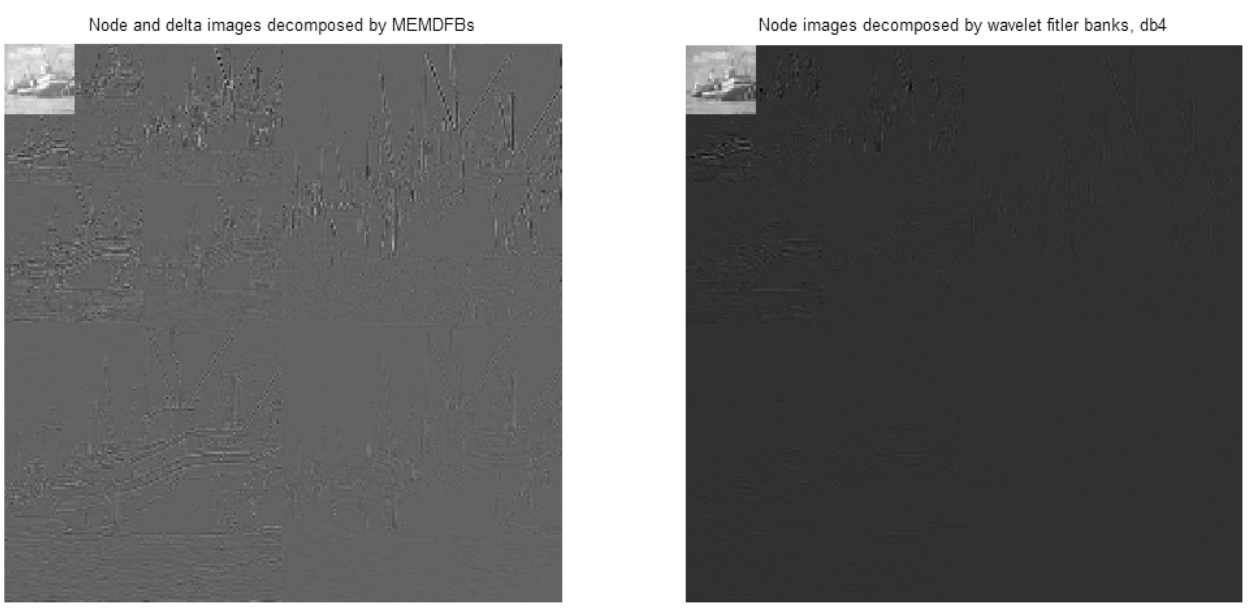

(c)

dee image at decomposition level 1

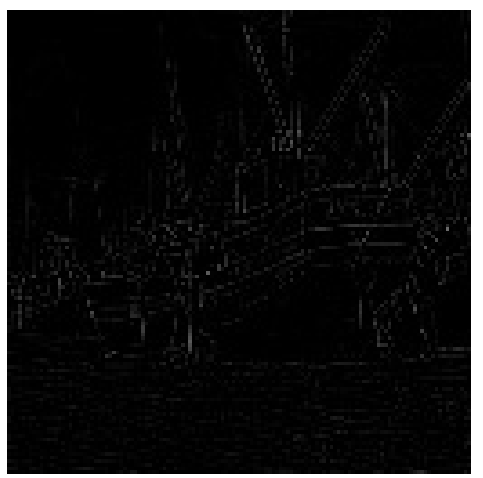

dee image at decomposition level 2

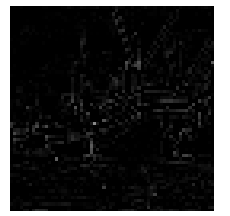

dee image at decomposition level 3

(d)

Fig. 10. (Continued)

each node matrix, $X_{i+1,2 j}$ and $X_{i+1,2 j+1}$, in Fig. 9(a) has three redundant images with pixels in the column, row, and diagonal directions, note that the node images of $X_{i+1,2 j}^{\mathrm{Img}}$ and $X_{i+1,2 j+1}^{\mathrm{Img}}$ in Fig. 10(c) are reconstructed from each node matrices by an arithmetic average of three images, given as

$$
\left\{\begin{array}{l}
X_{i+1,2 j}^{\operatorname{Img}}=\left(R_{c}^{e e}+R_{r}^{e e}+R_{d}^{e e}\right) / 3 \\
X_{i+1,2 j+1}^{\operatorname{Img}}=\left(I_{c}^{e e}+I_{r}^{e e}+I_{d}^{e e}\right) / 3 .
\end{array}\right.
$$

Those averaged node images are used in Fig. 10. Figure 10(b) shows an octave tree used for decimated MEMDFBs and subimages indices to make left-hand side image of Fig. 10(c). Figure 10(c) is a combined image by error images [i.e. error subimages changed from $\Delta_{i j}$ matrices in Fig. 9(a)] and one residual at each level. 
Again, since each error subimage of $\Delta_{i j}$ in Fig. 9(a) also has redundant images, those are arithmetically averaged and shown in Figs. 10(c) and 10(d). For instance, the error image at node $(1,0)$ denoted by $D_{10}^{e o}$ in Figs. $10(\mathrm{~b})$ and $10(\mathrm{~d})$ is obtained by $D_{10}^{e o}=\left(\Delta_{c}^{e o}+\Delta_{r}^{e o}+\Delta_{d}^{e o}\right) / 3$. All other error subimages in Figs. 10(b)-10(d) are obtained by the same manner as

$$
\left\{\begin{array}{l}
D_{i+1, j}^{o e}=\left(\Delta_{c}^{o e}+\Delta_{d}^{o e}+\Delta_{r}^{o e}\right) / 3 \\
D_{i+1, j}^{e o}=\left(\Delta_{c}^{e o}+\Delta_{d}^{e o}+\Delta_{r}^{e o}\right) / 3 \\
D_{i+1, j}^{o o}=\left(\Delta_{c}^{o o}+\Delta_{d}^{o o}+\Delta_{r}^{o o}\right) / 3
\end{array}\right.
$$

Note that one image of $D_{i j}^{o o}$ at each level is not included in right-hand side image of Fig. 10(b) and left-hand side image of Fig. 10(c). Those extra images of $D_{10}^{o o} \in R^{128 \times 128}, D_{20}^{o o} \in R^{64 \times 64}$, and $D_{30}^{o o} \in R^{32 \times 32}$ at each level - which are required for decimated MEMDFBs to achieve perfect reconstruction - are shown in Fig. 10(d). More details for subimages are also explained in Koh and RodriguezMarek [2013c] for single channel applications. Moreover, the right-hand side image on Fig. 10(c) shows the results of wavelet filter banks for the same end-nodes, where the mother wavelet of "db4" is used. Comparing the two images in Fig. 10(c), note that MEMDFBs show clearer directional edges in images of $(3,1),(2,1),(1,1)$ nodes, and $\Delta_{i j}^{o o}$ images. In other words, MEMDFBs shows clear vertical, horizontal, and diagonal edges, because of directional scanning of an image to apply MEMD. However, as shown in Fig. 10(d), it should be noted that one extra quarter size image is generated at each node in decimated MEMDFBs. The reason is explained in Koh and Rodriguez-Marek [2013c] for the univariate case. Wavelet filter banks show smaller variance in LH, HL, and HH subimages (i.e. good energy compaction in LL band), than those of MEMDFBs as shown in Fig. 10(c). A small variance in detail subimages (i.e. images on HL, LH, HH planes) is a good property for signal compression perspectives. As a comparison between decimated and undecimated MEMDFBs, note that all four images in Fig. 8 have the same size, $256 \times 256$ because there is no downsampling for undecimated MEMDFBs. Furthermore, note that any extra $\Delta_{i j}$ images are not required for undecimated MEMDFBs as shown in Fig. 8 because there is no downsampling involved.

All intermediate nodes in Fig. 9(a) are matrices having multichannels. Since the image size is getting reduced by factor of 4 at each decomposition level, each node signal (i.e. $X_{i, j}$ ) and error node signal (i.e. $\Delta_{i, j}$ ) have respectively $\frac{M N}{4^{i}} \times 3$ and $\frac{M N}{4^{i}} \times 9$ matrices at $i$ th decomposition level when it is applied to an original image of $M \times N$. In other words, each node and error signal in Fig. 9(a) is not an image but a matrix. However, all of intermediate node matrices in the analysis filter banks are not required for synthesis filter banks. Only end-node signals and $\Delta_{i j}$ s are required for perfect reconstruction at synthesis filter banks. Furthermore, all end-node signals in Fig. 9(a) have redundant data (i.e. redundant three channel subimages) because one image is scanned through the column, row, and diagonal directions. To keep only one subimage at each end-node like wavelet filter banks 


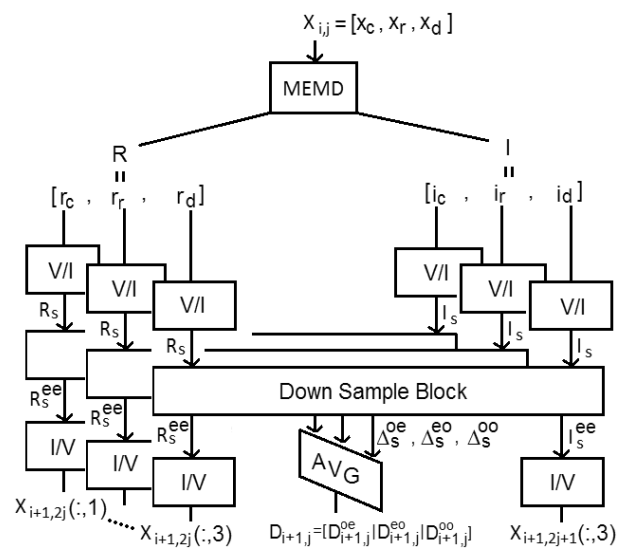

(a)

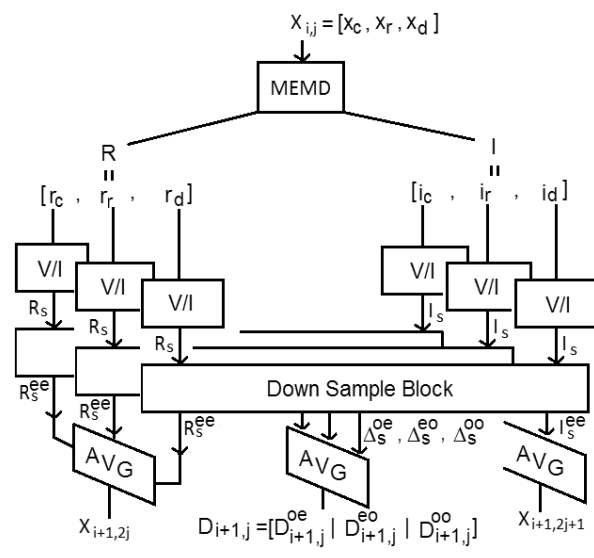

(b)

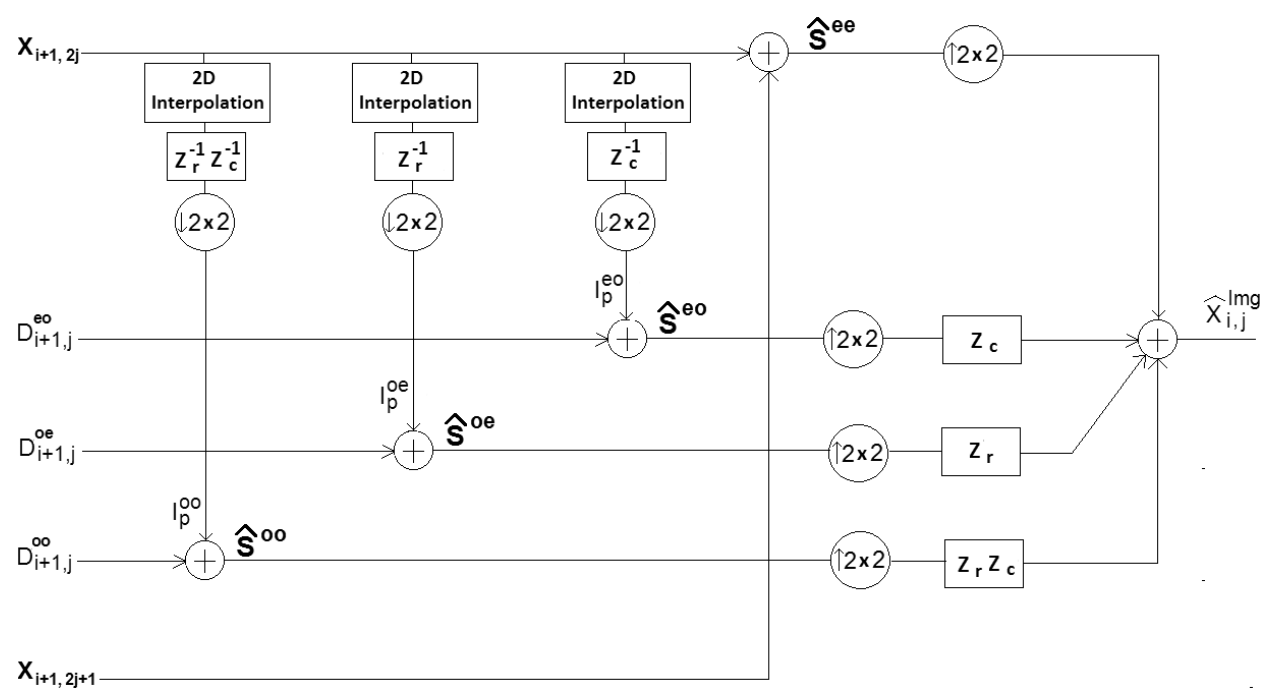

(c)

Fig. 11. (a) Intermediate nodes in decimated analysis MEMDFBs for image applications only, (b) end nodes in decimated analysis MEMDFBs for image applications only, (c) one stage for decimated synthesis MEMDFBs for image applications only.

(other intermediate nodes are fine with matrix form because they will not be kept and be used internally), the decimated EMDFBs in Figs. 9(a) and 9(c) needs a little revised form given in Fig. 11. First of all, DSB in Figs. 11(a) and 11(b) indicates Fig. 9(b). The "AVG" block does arithmetic average operation of three subimages in order to keep only one subimage and the AVG block is applied into end-nodes only. Since all intermediate nodes keep a matrix form, it uses " $I / V$ " block as shown in Fig. 11(a), which is for intermediate node only. For each end-node, only one 
subimage is kept as shown in Fig. 11(b), which is for end-node only. For instance, $X_{i+1,2 j}$ and $X_{i+1,2 j+1}$ node image in Fig. 11(b) is obtained by the average operation given in Eq. (2). Note in Fig. 11(b), that superscript "Img" to denote image form is dropped in node signal, $X_{i+1,2 j}$ and $X_{i+1,2 j+1}$, because the output of "AVG" block implies an image. And the averaged error images denoted by $D_{i+1, j}^{o e}, D_{i+1, j}^{e o}$, and $D_{i+1, j}^{o o}$ in Fig. 11 are also obtained by Eq. (3). Note that synthesis filter banks in Fig. 11(c) have the same structure of synthesis filter banks for 2D-EMD in Koh and Rodriguez-Marek [2013c] and it is re-drawn for further explanation of synthesis MEMDFBs. Figure 11 — which is for image applications only — provides all endnode and error subimages only with size of $\frac{M}{2^{i}} \times \frac{N}{2^{i}}$ at $i$ th decomposition level instead of end-node and error node matrices with size of $\frac{M N}{2^{i}} \times 3$ in Figs. 9(a) and 9 (c). Hence, summing up all end-node images of $X_{i, j}$ in Fig. 11(b) results in the same size of original given image like wavelet theory. However, note that decimated MEMDFBs in Fig. 11(b) need extra error-node images denoted by $D_{i, j} \in R^{\frac{M M}{2^{i}} \times \frac{3 N}{2^{i}}}$ at each ith decomposition level. Since all end-nodes and error-nodes have an image form, data reduction analysis of Fig. 11(b) is exactly same with that of Koh and Rodriguez-Marek [2013c]. Decimated MEMDFBs of Fig. 11(b) has about 1.33 times bigger size of decomposed data compared with traditional wavelet filter bank having an octave tree [Koh and Rodriguez-Marek (2013c)] because of extra error-node images. It can be analyzed like this: Since an octave filter banks have only one $D_{i j}$ at each decomposition level, it has total data size of $\left(\frac{M}{2^{P}} \times \frac{N}{2^{P}}\right)+\sum_{l=1}^{P}\left(\frac{M}{2^{l}} \times \frac{N}{2^{l}}\right)$ for node images, where the first term of $\left(\frac{M}{2^{P}} \times \frac{N}{2^{P}}\right)$ is for $X_{P, 0}$ node image (e.g. $X_{3,0}$ for $\left.P=3\right)$ and the second term of $\sum_{l=1}^{P}\left(\frac{M}{2^{t}} \times \frac{N}{2^{t}}\right)$ is to consider all $X_{p, 1}$ node imagesin an octave filter bank (e.g. $X_{1,1}, X_{2,1}$, and $X_{3,1}$ for $P=3$ ). The decimated MEMDFBs have additional error-node images of $\sum_{l=1}^{P}\left(\frac{M}{2^{l}} \times \frac{3 N}{2^{l}}\right)$ for $D_{i j}$. Hence, total size of decomposed node images and error-node images is

$$
\begin{aligned}
\left(\frac{M}{2^{P}} \times \frac{N}{2^{P}}\right)+\sum_{l=1}^{P}\left(\frac{M}{2^{l}} \times \frac{N}{2^{l}}\right)+\sum_{l=1}^{P}\left(\frac{M}{2^{l}} \times \frac{3 N}{2^{l}}\right) \\
=\frac{M N}{4^{P}}+\frac{M N}{3}\left(1-\frac{1}{4^{P}}\right)+M N\left(1-\frac{1}{4^{P}}\right) \\
=\frac{M N}{4^{P}}+\frac{4 M N}{3}\left(1-\frac{1}{4^{P}}\right) .
\end{aligned}
$$

Hence, total data size of decimated MEMDFBs of Figs. 11(a) and 11(c) for an octave filter banks for $P \geq 3$ decomposition level is $\approx 1.33$. For a full binary tree, decimated MEMDFBs of Fig. 11 need a total $M N\left(\frac{3}{2}-\frac{1}{2^{P+1}}\right)<\frac{3}{2} M N$ data, where $P$ is decomposition level for $M \times N$ image, it means decimated MEMDFBs need maximum 1.5 times bigger decomposed data compared with wavelet packets having full binary tree because wavelet packets have the size of $M N$ by totaling all decomposed images in fully binary tree nodes [Koh and Rodriguez-Marek (2013c)]. Although only end-node subimages (not matrices) and error-node subimages are considered 
in Fig. 11, the perfect reconstruction is also preserved using those subimages only. Perfect reconstruction of decimated MEMDFBs of Fig. 11 is formalized by following theorem (a detailed proof is given in Appendix B).

Theorem 3. With the same affine invariant interpolation in analysis and synthesis stages, the analysis and synthesis MEMDFBs in Fig. 11 form perfectly reconstructable filter banks, with aliasing cancelled.

\subsection{One-dimensional signals}

To apply the same idea of MEMDFBs in Figs. 4 and 6 to one-dimensional cases (e.g. speech and/or audio signals, etc.), NA-MEMD explained in previous section is used instead for the "MEMD" block in Fig. 4 because its mode-splitting property is better than EMD and/or EEMD as shown in Rehman et al. [2013]. These filter banks are coined NA-MEMDFBs and the results of decimated NA-MEMDFBs for a speech is shown in Fig. 12. Although two noise channels are used in NAMEMD, the NA-MEMD shows negligibly small errors in a reconstructed signal, because the noise channels are not directly added into a desired signal as explained in Rehman et al. [2013]. It results in a reconstructed signal having more than 300 [dB] SNR, which satisfies perfect reconstruction as well, as shown in Fig. 12(a).
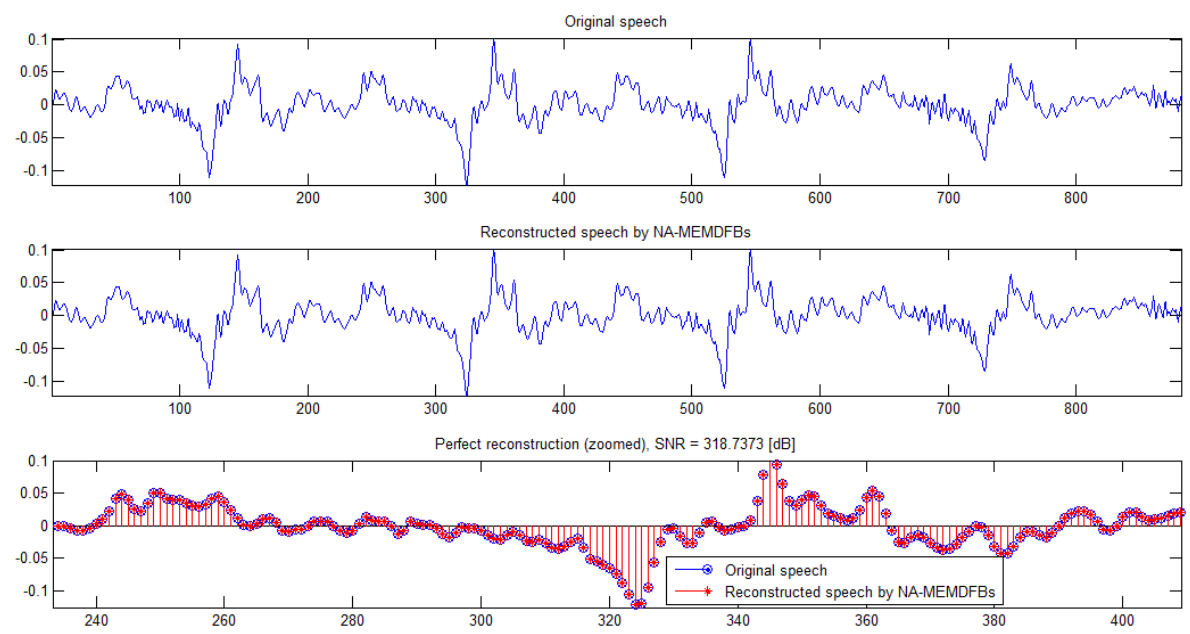

(a)

Fig. 12. Results of decimated NA-MEMDFBs for univariable applications. (a) Perfect reconstruction by decimated NA-MEMDFBs of $(6,0),(6,1),(5,1),(4,1),(3,1),(2,1)$, and $(1,1)$ end-nodes, (b) each node signal decomposed by decimated NA-MEMDFBs, where the last row through up to first row are respectively corresponding to $(6,0),(6,1),(5,1),(4,1),(3,1),(2,1)$, and $(1$, 1 ) end-nodes and (c) comparison of decimated NA-MEMDFBs with wavelet filter banks for the same octave tree, where the last row through up to first row are respectively corresponding to $(6$, $0),(6,1),(5,1),(4,1),(3,1),(2,1)$, and $(1,1)$ end-nodes. 


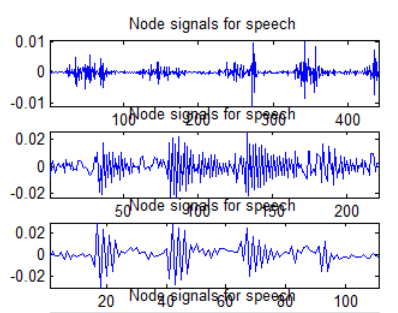

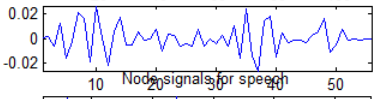
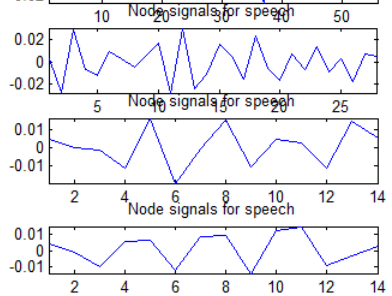
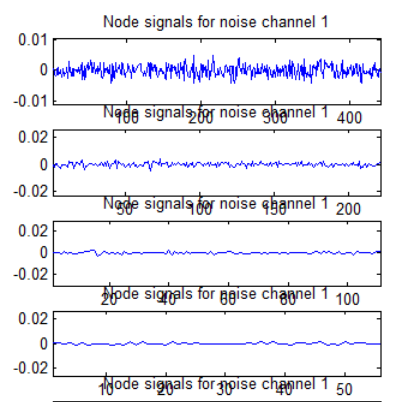

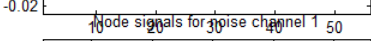
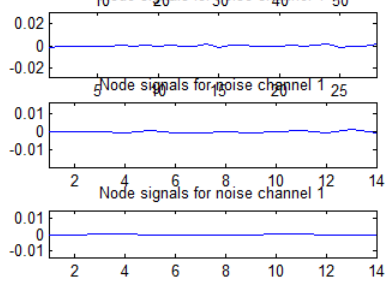
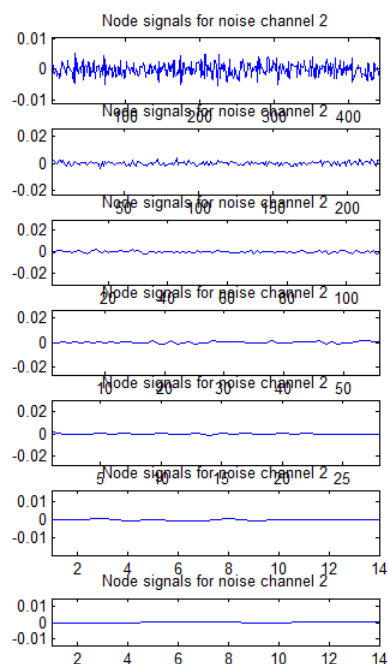

(b)
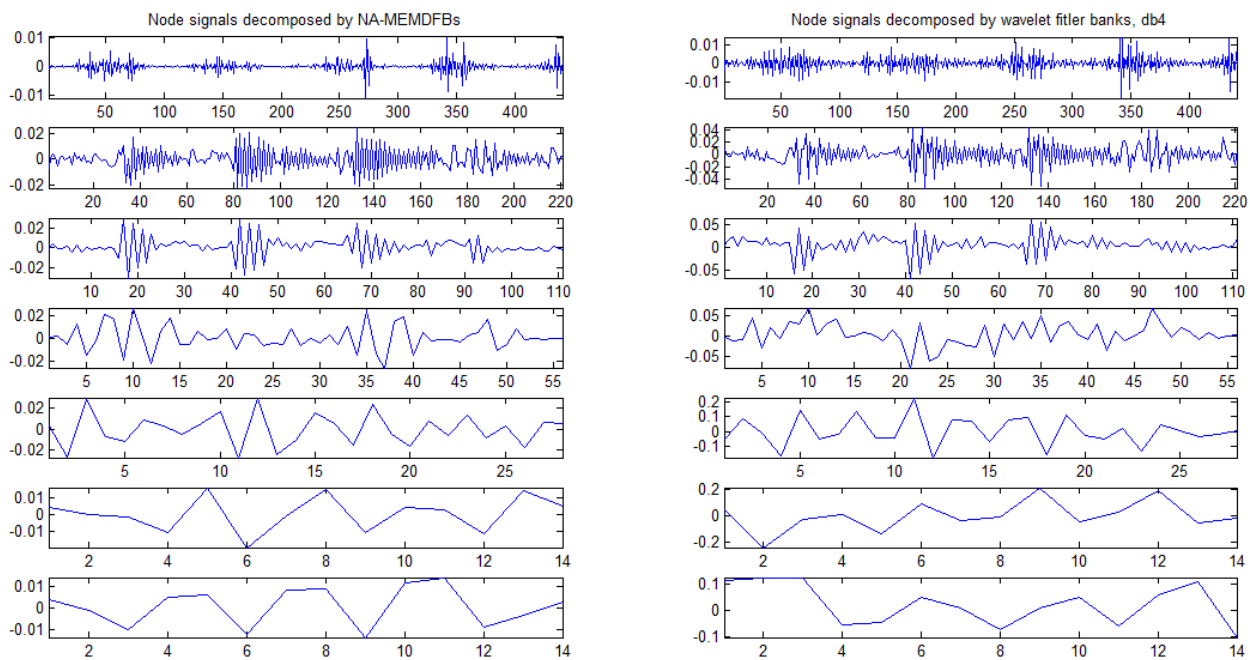

(c)

Fig. 12. (Continued)

Figure 12(b) shows each decomposed node signal for a speech and two noise channels, where two noise channels are discarded at the end because it only assists to MEMD for better mode-splitting with enough channels. Again, note that delta signals explained in Koh and Rodriguez-Marek [2013a, 2013b], which are required for perfect reconstruction, are not shown in Fig. 12(b). Figure 12(c) shows comparison between NA-MEMDFBs and wavelet filter banks for a speech, where an octave tree 

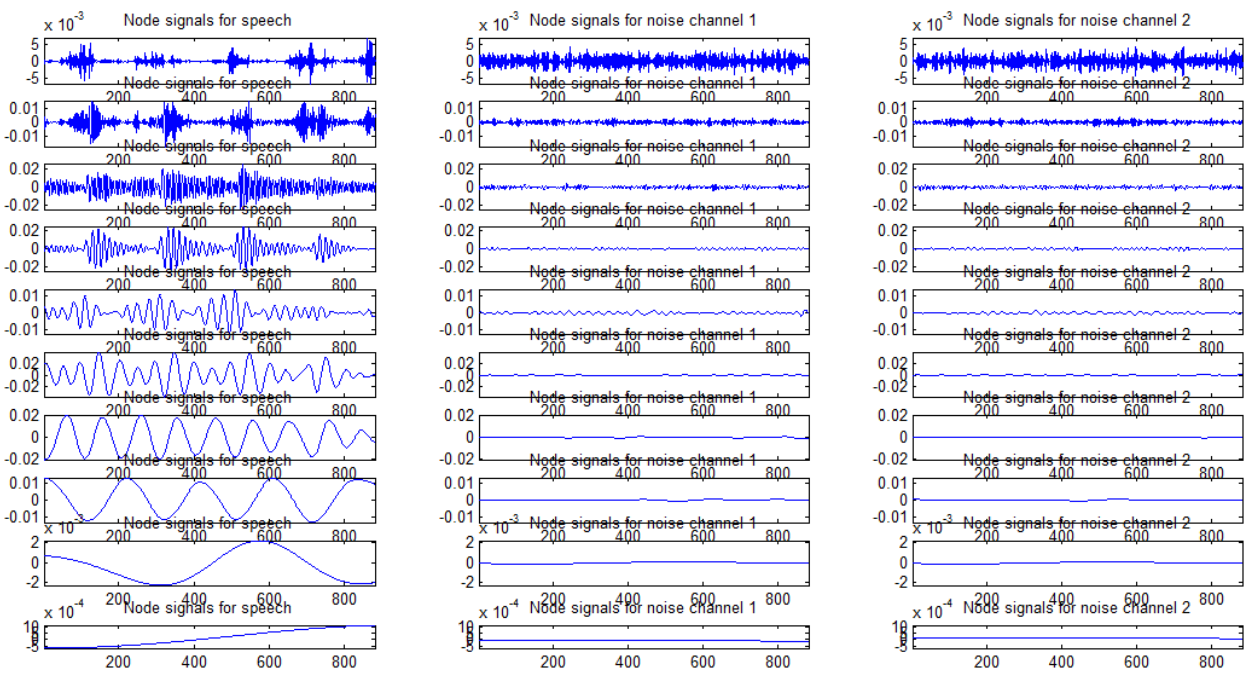

Fig. 13. Decomposed node signals of undecimated NA-MEMDFBs for univariable applications, where the last row through up to first row are respectively corresponding to $(9,0),(9,1),(8,1)$, $(7,1),(6,1),(5,1),(4,1),(3,1),(2,1)$, and $(1,1)$ end-nodes.

is applied. Decimated NA-MEMDFBs show clearer node signals at $(6,0),(6,1)$, and $(5,1)$ than those of wavelet filter banks, where "db4" mother wavelet is used. To obtain Fig. 12, two noise channels are used in NA-MEMD with SNR of 20 [dB] for an octave tree of $(6,0),(6,1),(5,1),(4,1),(3,1),(2,1)$ and $(1,1)$ end-nodes. Since there are not enough remaining samples after 6 th decomposition level (i.e. because of downsamplings), it shows only up to decomposition level of 6 . Figure 13 shows decomposed node signals for undecimated NA-MEMDFBs. Note that each node signal in undecimated NA-MEMDFBs has the same length as shown in Fig. 13, where the last row through up to first row are respectively corresponding to $(9,0)$, $(9,1),(8,1),(7,1),(6,1),(5,1),(4,1),(3,1),(2,1)$, and $(1,1)$ end-nodes.

Since the proposed decimated NA-MEMDFBs and MEMDFBs are applicable to any arbitrary nodes, they can be extended to NA-MEMD packets and MEMD packets, coined by the similar terminology as in wavelet theory. The undecimated NA-MEMDFBs and MEMDFBs have no signals in many nodes except for end-nodes in an octave tree as explained in previous section. Hence, when it is applied into any arbitrary nodes, it does not have meaningful MEMD packets, unlike wavelet packets. However, decimated NA-MEMD packets and MEMD packets lead to meaningful node signals because any node signal is usually available. As an example, each node signal decomposed by NA-MEMD packets is shown in Fig. 14(a) up to decomposition level of 3 for a speech signal. For comparison with wavelet packets, each node signal decomposed by wavelet packets with "db4" is shown in Fig. 14(b). Such MEMD packets can be applied to images and multivariate signals as well, because decimated MEMDFBs are applicable to arbitrary tree structures. 

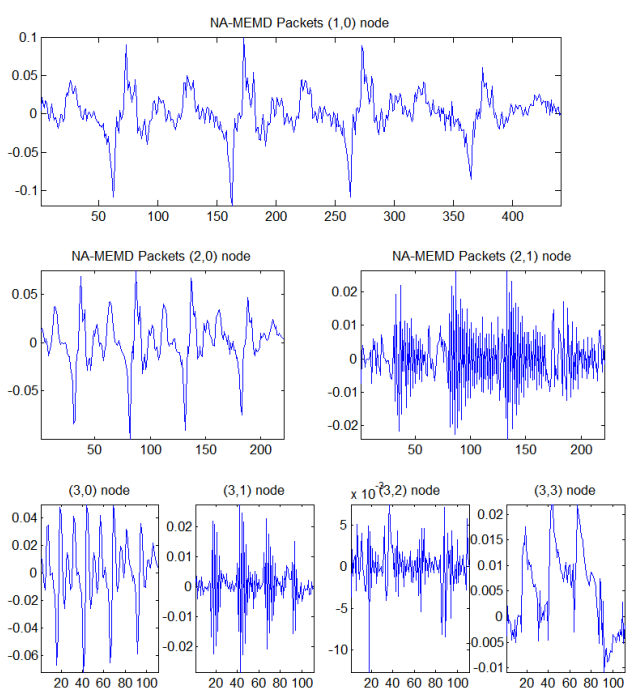

(a)
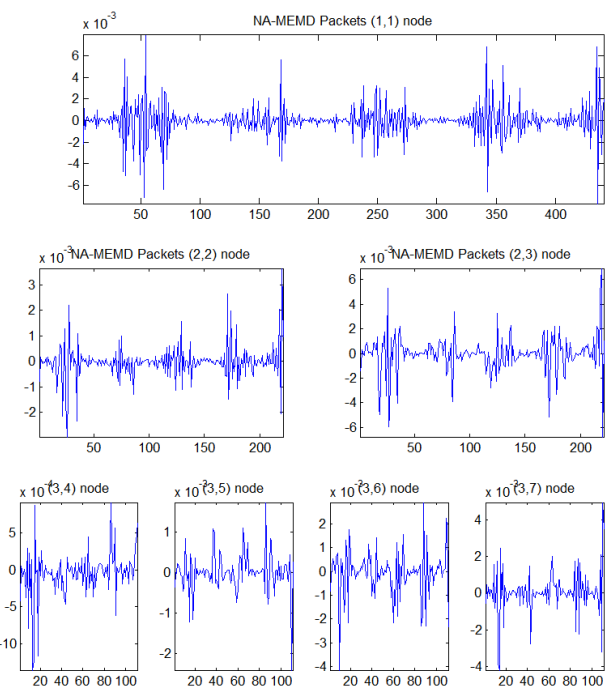

)
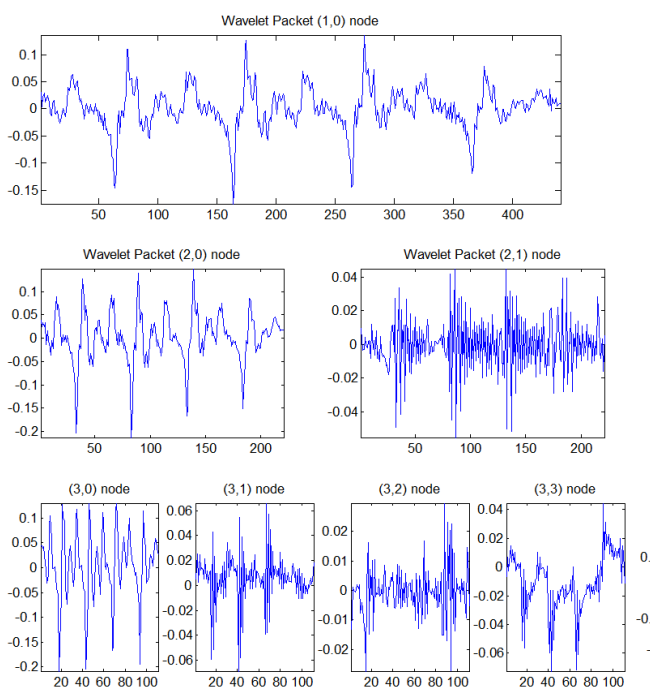
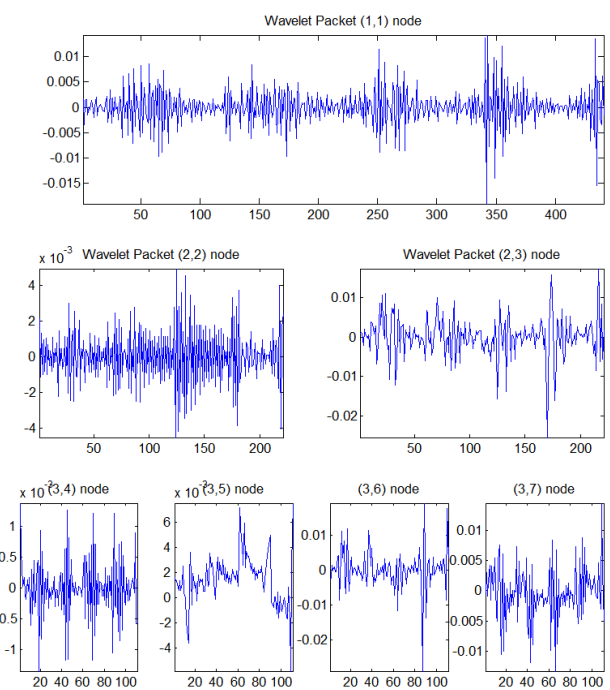

(b)

Fig. 14. Comparison of NA-MEMD Packets with wavelet packets for decomposition level 3 with "db4" for a speech signal. (a) Each node signal for NA-MEMD packets and (b) each node signal for wavelet packet.

\section{Conclusion}

Undecimated/decimated MEMDFBs and NA-MEMDFBs which can be applied to any arbitrary tree are developed in this paper for multivariate, speech/audio, and image applications. Decimated MEMDFBs and NA-MEMDFBs lead to perfect 
reconstruction with aliasing cancelled even without any traditional filters. For undecimated EMDFBs having an octave tree, it shows exactly the same results as those of original MEMD, because MEMD has good mode-splitting property. NA-MEMDFBs — which are developed for univariate applications - also lead to perfect reconstruction although noise channels are used. The new filter banks developed in this paper can handle nonlinear and nonstationary signals because they do not need any assumptions of linearity and/or stationary, which are mandatory assumptions in traditional filtering and filter banks. Furthermore, since the developed filter banks can be applied to any arbitrary trees, they are extended to undecimated/decimated NA-MEMD and MEMD packets. However, undecimated NA-MEMDFBs and MEMDFBs do not need any further decompositions from a given octave tree, unless there is any remaining mode in a node signal because of good mode-splitting property in MEMD and NA-MEMD. In addition, any node signals in decimated EMDFBs and NA-MEMD result in various options for flexible frequency bands, depending on the application.

\section{Appendix A. Proof for Theorem 2}

In Figs. 9(a) and 9(c), DSB and USB is applied identically for each column of $R=\left[r_{c}, r_{r}, r_{d}\right]$ and $I=\left[i_{c}, i_{r}, i_{d}\right]$ to recover one column input of $X_{i, j}=\left[x_{c}, x_{r}, x_{d}\right]$. Hence, let us consider only one general column vector denoted by $x_{s}$, where the subscript $s \in\{c, r, d\}$ and note $x_{s}=r_{s}+i_{s}$ by the nature of MEMD. The equation in column vectors are equivalent to $X_{s}=R_{s}+I_{s}$ with each corresponding subimage changed by $V / I$ block. If a same 2D-interpolation is used in DSB and USB of Figs. $9(\mathrm{~b})$ and $9(\mathrm{~d})$ respectively, then the estimated residual images denoted by $\hat{R}_{s}^{e o}, \hat{R}_{s}^{o e}$, and $\hat{R}_{s}^{o o}$ are same at DSB and USB in Figs. $9(\mathrm{~b})$ and $9(\mathrm{~d})$, respectively. Hence, $\hat{S}_{s}^{e o}=S_{s}^{e o}, \hat{S}_{s}^{o e}=S_{s}^{o e}$, and $\hat{S}_{s}^{o o}=S_{s}^{o o}$ are obtained at the USB. Let us denote $R_{s}^{e e}\left(z_{r}, z_{c}\right)$ implies two-dimensional $Z$ transform of $R_{s}^{e e}[m, n]$, which is a pixel at $(m, n)$ location, then the theorem is proved as follows:

Since internal signals of DSB in Fig. 9(b) are the case of rectangular decimation matrix of $M=\left[\begin{array}{ll}2 & 0 \\ 0 & 2\end{array}\right]$ [Vaidyanathan (1993)], all internal signals can be expressed as

$$
\begin{aligned}
R_{s}^{e e}\left(z_{r}, z_{c}\right)= & \frac{1}{4}\left\{R_{s}\left(z_{r}^{\frac{1}{2}}, z_{c}^{\frac{1}{2}}\right)+R_{s}\left(-z_{r}^{\frac{1}{2}}, z_{c}^{\frac{1}{2}}\right)+R_{s}\left(z_{r}^{\frac{1}{2}},-z_{c}^{\frac{1}{2}}\right)+R_{s}\left(-z_{r}^{\frac{1}{2}},-z_{c}^{\frac{1}{2}}\right)\right\} \\
R_{s}^{e o}\left(z_{r}, z_{c}\right)= & \frac{1}{4}\left\{z_{c}^{-\frac{1}{2}} R_{s}\left(z_{r}^{\frac{1}{2}}, z_{c}^{\frac{1}{2}}\right)+z_{c}^{-\frac{1}{2}} R_{s}\left(-z_{r}^{\frac{1}{2}}, z_{c}^{\frac{1}{2}}\right)-z_{c}^{-\frac{1}{2}} R_{s}\left(z_{r}^{\frac{1}{2}},-z_{c}^{\frac{1}{2}}\right)\right. \\
& \left.-z_{c}^{-\frac{1}{2}} R_{s}\left(-z_{r}^{\frac{1}{2}},-z_{c}^{\frac{1}{2}}\right)\right\} \\
R_{s}^{o e}\left(z_{r}, z_{c}\right)= & \frac{1}{4}\left\{z_{r}^{-\frac{1}{2}} R_{s}\left(z_{r}^{\frac{1}{2}}, z_{c}^{\frac{1}{2}}\right)-z_{r}^{-\frac{1}{2}} R_{s}\left(-z_{r}^{\frac{1}{2}}, z_{c}^{\frac{1}{2}}\right)+z_{r}^{-\frac{1}{2}} R_{s}\left(z_{r}^{\frac{1}{2}},-z_{c}^{\frac{1}{2}}\right)\right. \\
& \left.-z_{r}^{-\frac{1}{2}} R_{s}\left(-z_{r}^{\frac{1}{2}},-z_{c}^{\frac{1}{2}}\right)\right\}
\end{aligned}
$$




$$
\begin{aligned}
& R_{s}^{o o}\left(z_{r}, z_{c}\right)= \frac{1}{4}\left\{z_{r}^{-\frac{1}{2}} z_{c}^{-\frac{1}{2}} R_{s}\left(z_{r}^{\frac{1}{2}}, z_{c}^{\frac{1}{2}}\right)-z_{r}^{-\frac{1}{2}} z_{c}^{-\frac{1}{2}} R_{s}\left(-z_{r}^{\frac{1}{2}}, z_{c}^{\frac{1}{2}}\right)\right. \\
&\left.-z_{r}^{-\frac{1}{2}} z_{c}^{-\frac{1}{2}} R_{s}\left(z_{r}^{\frac{1}{2}},-z_{c}^{\frac{1}{2}}\right)+z_{r}^{-\frac{1}{2}} z_{c}^{-\frac{1}{2}} R_{s}\left(-z_{r}^{\frac{1}{2}},-z_{c}^{\frac{1}{2}}\right)\right\} \\
& I_{s}^{e e}\left(z_{r}, z_{c}\right)= \frac{1}{4}\left\{I_{s}\left(z_{r}^{\frac{1}{2}}, z_{c}^{\frac{1}{2}}\right)+I_{s}\left(-z_{r}^{\frac{1}{2}}, z_{c}^{\frac{1}{2}}\right)+I_{s}\left(z_{r}^{\frac{1}{2}},-z_{c}^{\frac{1}{2}}\right)+I_{s}\left(-z_{r}^{\frac{1}{2}},-z_{c}^{\frac{1}{2}}\right)\right\} \\
& I_{s}^{e o}\left(z_{r}, z_{c}\right)= \frac{1}{4}\left\{z_{c}^{-\frac{1}{2}} I_{s}\left(z_{r}^{\frac{1}{2}}, z_{c}^{\frac{1}{2}}\right)+z_{c}^{-\frac{1}{2}} I_{s}\left(-z_{r}^{\frac{1}{2}}, z_{c}^{\frac{1}{2}}\right)-z_{c}^{-\frac{1}{2}} I_{s}\left(z_{r}^{\frac{1}{2}},-z_{c}^{\frac{1}{2}}\right)\right. \\
&\left.-z_{c}^{-\frac{1}{2}} I_{s}\left(-z_{r}^{\frac{1}{2}},-z_{c}^{\frac{1}{2}}\right)\right\} \\
& I_{s}^{o e}\left(z_{r}, z_{c}\right)= \frac{1}{4}\left\{z_{r}^{-\frac{1}{2}} I_{s}\left(z_{r}^{\frac{1}{2}}, z_{c}^{\frac{1}{2}}\right)-z_{r}^{-\frac{1}{2}} I_{s}\left(-z_{r}^{\frac{1}{2}}, z_{c}^{\frac{1}{2}}\right)+z_{r}^{-\frac{1}{2}} I_{s}\left(z_{r}^{\frac{1}{2}},-z_{c}^{\frac{1}{2}}\right)\right. \\
&\left.-z_{r}^{-\frac{1}{2}} I_{s}\left(-z_{r}^{\frac{1}{2}},-z_{c}^{\frac{1}{2}}\right)\right\} \\
& \frac{1}{4}\left\{z_{r}^{-\frac{1}{2}} z_{c}^{-\frac{1}{2}} I_{s}\left(z_{r}^{\frac{1}{2}}, z_{c}^{\frac{1}{2}}\right)-z_{r}^{-\frac{1}{2}} z_{c}^{-\frac{1}{2}} I_{s}\left(-z_{r}^{\frac{1}{2}}, z_{c}^{\frac{1}{2}}\right)\right. \\
&\left.-z_{r}^{-\frac{1}{2}} z_{c}^{-\frac{1}{2}} I_{s}\left(z_{r}^{\frac{1}{2}},-z_{c}^{\frac{1}{2}}\right)+z_{r}^{-\frac{1}{2}} z_{c}^{-\frac{1}{2}} I_{s}\left(-z_{r}^{\frac{1}{2}},-z_{c}^{\frac{1}{2}}\right)\right\} . \\
& I_{s}^{o o}\left(z_{r}, z_{c}\right)
\end{aligned}
$$

Then, the recovered signal, $\hat{X}_{s}$, in USB is expressed as

$$
\begin{aligned}
\hat{X}_{s}\left(z_{r}, z_{c}\right)= & \hat{S}_{s}^{e e}\left(z_{r}^{2}, z_{c}^{2}\right)+z_{c} \hat{S}_{s}^{e o}\left(z_{r}^{2}, z_{c}^{2}\right)+z_{r} \hat{S}_{s}^{o e}\left(z_{r}^{2}, z_{c}^{2}\right)+z_{r} z_{c} \hat{S}_{s}^{o o}\left(z_{r}^{2}, z_{c}^{2}\right) \\
= & \frac{1}{4}\left\{R_{s}\left(z_{r}, z_{c}\right)+I_{s}\left(z_{r}, z_{c}\right)+R_{s}\left(-z_{r}, z_{c}\right)+I_{s}\left(-z_{r}, z_{c}\right)\right. \\
& \left.+R_{s}\left(z_{r},-z_{c}\right)+I_{s}\left(z_{r},-z_{c}\right)+R_{s}\left(-z_{r},-z_{c}\right)+I_{s}\left(-z_{r},-z_{c}\right)\right\} \\
& +\frac{1}{4}\left\{R_{s}\left(z_{r}, z_{c}\right)+I_{s}\left(z_{r}, z_{c}\right)+R_{s}\left(-z_{r}, z_{c}\right)+I_{s}\left(-z_{r}, z_{c}\right)\right. \\
& \left.-R_{s}\left(z_{r},-z_{c}\right)-I_{s}\left(z_{r},-z_{c}\right)-R_{s}\left(-z_{r},-z_{c}\right)-I_{s}\left(-z_{r},-z_{c}\right)\right\} \\
& +\frac{1}{4}\left\{R_{s}\left(z_{r}, z_{c}\right)+I_{s}\left(z_{r}, z_{c}\right)-R_{s}\left(-z_{r}, z_{c}\right)-I_{s}\left(-z_{r}, z_{c}\right)\right. \\
& \left.+R_{s}\left(z_{r},-z_{c}\right)+I_{s}\left(z_{r},-z_{c}\right)-R_{s}\left(-z_{r},-z_{c}\right)-I_{s}\left(-z_{r},-z_{c}\right)\right\} \\
& +\frac{1}{4}\left\{R_{s}\left(z_{r}, z_{c}\right)+I_{s}\left(z_{r}, z_{c}\right)-R_{s}\left(-z_{r}, z_{c}\right)-I_{s}\left(-z_{r}, z_{c}\right)\right. \\
& \left.-R_{s}\left(z_{r},-z_{c}\right)-I_{s}\left(z_{r},-z_{c}\right)+R_{s}\left(-z_{r},-z_{c}\right)+I_{s}\left(-z_{r},-z_{c}\right)\right\} \\
& \frac{1}{4}\left\{4 R_{s}\left(z_{r}, z_{c}\right)+4 I_{s}\left(z_{r}, z_{c}\right)\right\}=R_{s}\left(z_{r}, z_{c}\right)+I_{s}\left(z_{r}, z_{c}\right) \\
= & X_{s}\left(z_{r}, z_{c}\right), \quad \forall s \in\{c, r, d\},
\end{aligned}
$$


where each subimages of $\hat{S}_{s}^{e o}=S_{s}^{e o}, \hat{S}_{s}^{o e}=S_{s}^{o e}$, and $\hat{S}_{s}^{o o}=S_{s}^{o o}$ are expressed as

$$
\begin{aligned}
& \hat{S}_{s}^{e e}\left(z_{r}^{2}, z_{c}^{2}\right)=R_{s}^{e e}\left(z_{r}^{2}, z_{c}^{2}\right)+I_{s}^{e e}\left(z_{r}^{2}, z_{c}^{2}\right) \\
& =\frac{1}{4}\left\{R_{s}\left(z_{r}, z_{c}\right)+R_{s}\left(-z_{r}, z_{c}\right)+R_{s}\left(z_{r},-z_{c}\right)+R_{s}\left(-z_{r},-z_{c}\right)\right\} \\
& +\frac{1}{4}\left\{I_{s}\left(z_{r}, z_{c}\right)+I_{s}\left(-z_{r}, z_{c}\right)+I_{s}\left(z_{r},-z_{c}\right)+I_{s}\left(-z_{r},-z_{c}\right)\right\}, \\
& \hat{S}_{s}^{e o}\left(z_{r}^{2}, z_{c}^{2}\right)=R_{s}^{e o}\left(z_{r}^{2}, z_{c}^{2}\right)+I_{s}^{e o}\left(z_{r}^{2}, z_{c}^{2}\right) \\
& =\frac{1}{4}\left\{z_{c}^{-1} R_{s}\left(z_{r}, z_{c}\right)+z_{c}^{-1} R_{s}\left(-z_{r}, z_{c}\right)\right. \\
& \left.-z_{c}^{-1} R_{s}\left(z_{r},-z_{c}\right)-z_{c}^{-1} R_{s}\left(-z_{r},-z_{c}\right)\right\} \\
& +\frac{1}{4}\left\{z_{c}^{-1} I_{s}\left(z_{r}, z_{c}\right)+z_{c}^{-1} I_{s}\left(-z_{r}, z_{c}\right)\right. \\
& \left.-z_{c}^{-1} I_{s}\left(z_{r},-z_{c}\right)-z_{c}^{-1} I_{s}\left(-z_{r},-z_{c}\right)\right\}, \\
& \hat{S}_{s}^{o e}\left(z_{r}^{2}, z_{c}^{2}\right)=R_{s}^{o e}\left(z_{r}^{2}, z_{c}^{2}\right)+I_{s}^{o e}\left(z_{r}^{2}, z_{c}^{2}\right) \\
& =\frac{1}{4}\left\{z_{r}^{-1} R_{s}\left(z_{r}, z_{c}\right)-z_{r}^{-1} R_{s}\left(-z_{r}, z_{c}\right)\right. \\
& \left.+z_{r}^{-1} R_{s}\left(z_{r},-z_{c}\right)-z_{r}^{-1} R_{s}\left(-z_{r},-z_{c}\right)\right\} \\
& +\frac{1}{4}\left\{z_{r}^{-1} I_{s}\left(z_{r}, z_{c}\right)-z_{r}^{-1} I_{s}\left(-z_{r}, z_{c}\right)\right. \\
& \left.+z_{r}^{-1} I_{s}\left(z_{r},-z_{c}\right)-z_{r}^{-1} I_{s}\left(-z_{r},-z_{c}\right)\right\}, \\
& \hat{S}_{s}^{o o}\left(z_{r}^{2}, z_{c}^{2}\right)=R_{s}^{o o}\left(z_{r}^{2}, z_{c}^{2}\right)+I_{s}^{o o}\left(z_{r}^{2}, z_{c}^{2}\right) \\
& =\frac{1}{4}\left\{z_{r}^{-1} z_{c}^{-1} R_{s}\left(z_{r}, z_{c}\right)-z_{r}^{-1} z_{c}^{-1} R_{s}\left(-z_{r}, z_{c}\right)\right. \\
& \left.-z_{r}^{-1} z_{c}^{-1} R_{s}\left(z_{r},-z_{c}\right)+z_{r}^{-1} z_{c}^{-1} R_{s}\left(-z_{r},-z_{c}\right)\right\} \\
& +\frac{1}{4}\left\{z_{r}^{-1} z_{c}^{-1} I_{s}\left(z_{r}, z_{c}\right)-z_{r}^{-1} z_{c}^{-1} I_{s}\left(-z_{r}, z_{c}\right)\right. \\
& \left.-z_{r}^{-1} z_{c}^{-1} I_{s}\left(z_{r},-z_{c}\right)+z_{r}^{-1} z_{c}^{-1} I_{s}\left(-z_{r},-z_{c}\right)\right\} .
\end{aligned}
$$

Note that the reconstructed $\hat{X}_{s}[m, n]$ node image corresponding to $\hat{X}_{s}\left(z_{r}, z_{c}\right)$ is changed into a column vector of $x_{c}, x_{r}$ or $x_{d}$ depending on subscript $s \in\{c, r, d\}$ by the " $I / V$ " block without any data loss because each block denoted by " $I / V$ " and " $V / I$ " does simple format change from vector to image or vice versa. Since 
there is no data loss in the blocks of " $I / V$ " and " $V / I$ ", perfect reconstruction of overall systems of Figs. 9(a) and 9(c) is achieved if the pair of DSB and USB leads to perfect reconstruction. This was shown in Koh and Rodriguez-Marek [2013c] for a single channel case by intuition without rigorous proof.

\section{Appendix B. Proof for Theorem 3}

In this Appendix, Theorem 3, which guarantees the perfect reconstruction of image applications of MEMDFBs, is proved. To begin with, an affine invariant interpolation is defined as follows, where it uses the same concept of the affine invariance in Farin [2002].

Definition: (Affine invariant interpolation): For an interpolation of an image $S$ denoted by $\mathrm{I}_{p}(S)$, an affine invariant interpolation is defined as

$$
I_{p}\left(\sum_{k=1}^{P} a_{k} S_{k}\right)=\sum_{k=1}^{P} a_{k} I_{p}\left(S_{k}\right), \quad \text { where } \sum_{k=1}^{P} a_{k}=1 .
$$

To prove Theorem 3, note in Figs. 11(a) and 11(b) that, as Eq. (1), $X_{i j}^{\mathrm{Img}}=$ $\left(X_{i j, c}^{\mathrm{Img}}+X_{i j, r}^{\mathrm{Img}}+X_{i j, d}^{\mathrm{Img}}\right) / 3$ is an averaged image corresponding to $X_{i j} \in R^{L \times 3}$, where $X_{i j, c}^{\mathrm{img}}, X_{i j, r}^{\mathrm{img}}$, and $X_{i j, d}^{\mathrm{img}}$ are images changed respectively from $x_{c}, x_{r}$, and $x_{d}$ column vectors in node matrix, $X_{i, j}$. Since an image can be equivalently expressed by four subimages depending on pixel locations, each image is expressed with four subimages of $S_{s}^{e e}, S_{s}^{o e}, S_{s}^{e o}$, and $S_{s}^{o o}$ respectively depending on (even, even), (odd, even), (even, odd), and (odd, odd) row and column pixel indices for any subscript $s \in\{c, r, d\}$. In other words

$$
X_{i j, c}^{\operatorname{Img}}=\left[\begin{array}{cc}
S_{c}^{e e} & S_{c}^{o e} \\
S_{c}^{e o} & S_{c}^{o o}
\end{array}\right], \quad X_{i j, r}^{\operatorname{Img}}=\left[\begin{array}{cc}
S_{r}^{e e} & S_{r}^{o e} \\
S_{r}^{e o} & S_{r}^{o o}
\end{array}\right], \quad X_{i j, d}^{\operatorname{Img}}=\left[\begin{array}{cc}
S_{d}^{e e} & S_{d}^{o e} \\
S_{d}^{e o} & S_{d}^{o o}
\end{array}\right],
$$

where $S_{s}^{e e}=R_{s}^{e e}+I_{s}^{e e}, S_{s}^{o e}=R_{s}^{o e}+I_{s}^{o e}, S_{s}^{e o}=R_{s}^{e o}+I_{s}^{e o}$, and $S_{s}^{o o}=R_{s}^{o o}+$ $I_{s}^{o o}$ because of MEMD nature for any subscript $s \in\{c, r, d\}$. Hence, averaged image corresponding to $X_{i, j}=\left[x_{c}, x_{r}, x_{d}\right]$ node matrix in Figs. 11(a) and 11(b) is expressed by

$$
\begin{aligned}
X_{i j}^{\mathrm{Img}} & =\frac{1}{3}\left(X_{i j, c}^{\mathrm{Img}}+X_{i j, r}^{\mathrm{Img}}+X_{i j, d}^{\mathrm{Img}}\right) \\
& =\frac{1}{3}\left[\begin{array}{ll}
S_{c}^{e e}+S_{r}^{e e}+S_{d}^{e e} & S_{c}^{o e}+S_{r}^{o e}+S_{d}^{o e} \\
S_{c}^{e o}+S_{r}^{e o}+S_{d}^{e o} & S_{c}^{o o}+S_{r}^{o o}+S_{d}^{o o}
\end{array}\right] .
\end{aligned}
$$

Consider a same affine invariant interpolation for 2D-interplation blocks in Fig. 11(c) and in DSB of analysis MEMDFB of Figs. 11(a) and 11(b), then Theorem 3 is proved by starting from $\hat{X}_{i, j}^{\mathrm{Img}}$ in synthesis MEMDFB of Fig. 11(c) and getting to $X_{i j}^{\mathrm{Img}}$ in analysis MEMDFB of Fig. 11(b). In this proof, note that the 
blocks of $\uparrow 2 \times 2$ and $\downarrow 2 \times 2$ with shift operator of $z_{r}$ and $z_{c}$ are skipped because those blocks only related to weaving pixels into correct locations internally as shown in Appendix A

$$
\begin{aligned}
& \hat{X}_{i j}^{\operatorname{Img}}=\left[\begin{array}{cc}
\hat{S}^{e e} & \hat{S}^{o e} \\
\hat{S}^{e o} & \hat{S}^{o o}
\end{array}\right] \quad \text { with four subimages in Fig. 11(c). } \\
& \hat{X}_{i j}^{\mathrm{Img}}=\left[\begin{array}{ll}
X_{i+1,2 j}+X_{i+1,2 j+1} & D_{i+1, j}^{o e}+I_{p}^{o e}\left(X_{i+1,2 j}\right) \\
D_{i+1, j}^{e o}+I_{p}^{e o}\left(X_{i+1,2 j}\right) & D_{i+1, j}^{o o}+I_{p}^{o o}\left(X_{i+1,2 j}\right)
\end{array}\right] \quad \text { from Fig. 11(c), }
\end{aligned}
$$

where $I_{p}^{o e}\left(X_{i+1,2 j}\right)$ implies (odd, even) indexed samples from the 2D-interpolated image of $X_{i+1,2 j}$. Also, note that each node signal in Fig. 11(c) is an image by average operation, the superscript "Img" is dropped. Using Eq. (2), the following equation is obtained:

$$
\hat{X}_{i j}^{\mathrm{Img}}=\left[\begin{array}{c}
\left(R_{c}^{e e}+R_{r}^{e e}+R_{d}^{e e}\right) / 3+\left(I_{c}^{e e}+I_{r}^{e e}+I_{d}^{e e}\right) / 3 \\
D_{i+1, j}^{o e}+I_{p}^{o e}\left(\left\{R_{c}^{e e}+R_{r}^{e e}+R_{d}^{e e}\right\} / 3\right) \\
D_{i+1, j}^{e o}+I_{p}^{e o}\left(\left\{R_{c}^{e e}+R_{r}^{e e}+R_{d}^{e e}\right\} / 3\right) \\
D_{i+1, j}^{o o}+I_{p}^{o o}\left(\left\{R_{c}^{e e}+R_{r}^{e e}+R_{d}^{e e}\right\} / 3\right)
\end{array}\right] .
$$

Considering any affine invariant interpolation (e.g. linear interpolation, etc.), the following equation is obtained

$$
\hat{X}_{i j}^{\mathrm{Img}}=\left[\begin{array}{c}
\left(R_{c}^{e e}+R_{r}^{e e}+R_{d}^{e e}\right) / 3+\left(I_{c}^{e e}+I_{r}^{e e}+I_{d}^{e e}\right) / 3 \\
D_{i+1, j}^{o e}+\frac{1}{3}\left\{I_{p}^{o e}\left(R_{c}^{e e}\right)+I_{p}^{o e}\left(R_{r}^{e e}\right)+I_{p}^{o e}\left(R_{d}^{e e}\right)\right\} \\
D_{i+1, j}^{e o}+\frac{1}{3}\left\{I_{p}^{e o}\left(R_{c}^{e e}\right)+I_{p}^{e o}\left(R_{r}^{e e}\right)+I_{p}^{e o}\left(R_{d}^{e e}\right)\right\} \\
D_{i+1, j}^{o o}+\frac{1}{3}\left\{I_{p}^{o o}\left(R_{c}^{e e}\right)+I_{p}^{o o}\left(R_{r}^{e e}\right)+I_{p}^{o o}\left(R_{d}^{e e}\right)\right\}
\end{array}\right] .
$$

Using Eq. (3), the following equation is obtained:

$$
\hat{X}_{i j}^{\mathrm{Img}}=\left[\begin{array}{c}
\left(R_{c}^{e e}+R_{r}^{e e}+R_{d}^{e e}\right) / 3+\left(I_{c}^{e e}+I_{r}^{e e}+I_{d}^{e e}\right) / 3 \\
\left(\Delta_{c}^{o e}+\Delta_{d}^{o e}+\Delta_{r}^{o e}\right) / 3+\left\{I_{p}^{o e}\left(R_{c}^{e e}\right)+I_{p}^{o e}\left(R_{r}^{e e}\right)+I_{p}^{o e}\left(R_{d}^{e e}\right)\right\} / 3 \\
\left(\Delta_{c}^{e o}+\Delta_{d}^{e o}+\Delta_{r}^{e o}\right) / 3+\left\{I_{p}^{e o}\left(R_{c}^{e e}\right)+I_{p}^{e o}\left(R_{r}^{e e}\right)+I_{p}^{e o}\left(R_{d}^{e e}\right)\right\} / 3 \\
\left(\Delta_{c}^{o o}+\Delta_{d}^{o o}+\Delta_{r}^{o o}\right) / 3+\left\{I_{p}^{o o}\left(R_{c}^{e e}\right)+I_{p}^{o o}\left(R_{r}^{e e}\right)+I_{p}^{o o}\left(R_{d}^{e e}\right)\right\} / 3
\end{array}\right] .
$$

Since $\Delta_{s}^{o e}+I_{p}^{o e}\left(R_{s}^{e e}\right)=\Delta_{s}^{o e}+\hat{R}_{s}^{o e}=S_{s}^{o e}$ at DSB of Fig. 11(b) as shown in Fig. 9(b) and $R_{s}^{e e}+I_{s}^{e e}=S_{s}^{e e}$ for any subscript $s \in\{c, r, d\}$, the following equation 
is obtained:

$$
\begin{aligned}
\hat{X}_{i j}^{\operatorname{Img}} & =\left[\begin{array}{ll}
\frac{1}{3}\left\{S_{c}^{e e}+S_{r}^{e e}+S_{d}^{e e}\right\} & \frac{1}{3}\left\{S_{c}^{o e}+S_{r}^{o e}+S_{d}^{o e}\right\} \\
\frac{1}{3}\left\{S_{c}^{e o}+S_{r}^{e o}+S_{d}^{e o}\right\} & \frac{1}{3}\left\{S_{c}^{o o}+S_{r}^{o o}+S_{d}^{o o}\right\}
\end{array}\right] \\
= & \frac{1}{3}\left(X_{i j, c}^{\mathrm{Img}}+X_{i j, r}^{\mathrm{Img}}+X_{i j, d}^{\mathrm{Img}}\right)=X_{i j}^{\mathrm{Img}} \quad \text { from Eq. (B.2). }
\end{aligned}
$$

In other words, keeping only end-node images and error-node images also makes perfect reconstruction. Hence, decimated MEMDFBs in Fig. 11 also satisfy perfect reconstruction as long as the same affine invariant interpolation (e.g. linear interpolation, etc.) is used in analysis and synthesis of MEMDFBs.

\section{References}

Chatlani, N. and Soraghan, J. J. (2012). EMD-based filtering (EMDF) of low-frequency noise for speech enhancement. IEEE Trans. Audio, Speech Lang. Process., 20: 11581166, doi: 10.1109/TASL.2011.2172428.

Farin, G. (2002). Curves and Surfaces for CAGD: A Practical Guide., 5th edn. (Morgan Kaufmann Publishers, San Francisco, CA).

Huang, N. E., Shen, Z., Long, S., Wu, M., Shih, H., Zheng, Q., Yen, N., Tung, C. and Liu, H. (1998). The empirical mode decomposition and Hilbert spectrum for nonlinear and non-stationary time series analysis. Proc. R. Soc. Lond. A, 454: 903-995, doi:10.1098/rspa.1998.0193.

Koh, M. S. and Rodriguez-Marek, E. (2013a). Perfect reconstructable decimated onedimensional empirical mode decomposition filter banks. In The 3rd International Conference on Signal, Image Processing and Applications (ICSIA). Barcelona, Spain, August, 2013.

Koh, M. S. and Rodriguez-Marek, E. (2013b). Undecimated and decimated EMD nonuniform filterbanks approximating critical bands. In IASTED Signal Processing, Pattern Recognition and Applications (SPPRA). Innsbruck, Austria, February, 2013.

Koh, M. S. and Rodriguez-Marek, E. (2013c). Perfect reconstructable decimated twodimensional empirical mode decomposition filter banks. In IEEE International Conference on Acoustics, Speech, and Signal Processing (ICASSP). Vancouver, Canada, May, 2013.

Mandic, D. P., Rehman, N. U., Wu, Z. and Huang, N. E. (2013). Empirical mode decomposition based time-frequency analysis of multivariate signals. IEEE Signal. Process. Mag., 30: 74-86.

Omitaoumu, O. A., Protopopescu, V. A. and Ganguly, A. R. (2011). Empirical mode decomposition technique with conditional mutual information for denoising operational sensor data. IEEE Sens. J., 11: 2565-2575, doi: 10.1109/JSEN.2011.2142302.

Park, C., Looney, D., Kidmose, P., Ungstrup, M. and Mandic, D. P. (2011). Time-frequency analysis of EEG asymmetry using bivariate empirical mode decomposition. IEEE Trans. Neural Syst. Rehabil. Eng., 19: 366-373, doi: 10.1109/TNSRE.2011.2116805.

Rehman, N. U. and Mandic, D. P. (2010). Multivariate empirical mode decomposition. Proc. R. Soc. A, 466: 1291-1302.

Rehman, N. U. and Mandic, D. P. (2011). Filterbank property of multivariate EMD. IEEE Trans. Signal Process., 59: 2421-2426, doi: 10.1109/TSP.2011.2106779. 
Rehman, N. U., Park, C., Huang, N. E. and Mandic, D. P. (2013). EMD via MEMD: Multivariate noise-aided computation of standard EMD. Adv. Adapt. Data Anal., 5: 1-25, doi: 10.1142/S1793536913500076.

Vaidyanathan, P. P. (1993). Multirate Systems and Filter Banks (Prentice Hall Inc., Upper Saddle River, NJ).

Vetterli, M. and Kovacevic, J. (1995). Wavelets and Subband Coding (Prentice Hall PTR, Englewood Cliffs, NJ).

Wu, Z. and Huang, N. E. (2009). Ensemble empirical mode decomposition: A noise-assisted data analysis method. Adv. Adapt. Data Anal., 1: 1-41, doi: 10.1142/S179353690 9000047.

Zhang, R. R., Ma, S., Safak, E. and Hartzell, S. (2003). Hilbert-Huang transform analysis of dynamic and earthquake motion recordings. J. Eng. Mech., 129: 861-875, doi: 10.1061/(ASCE)0733-9399(2003)129:8(861). 